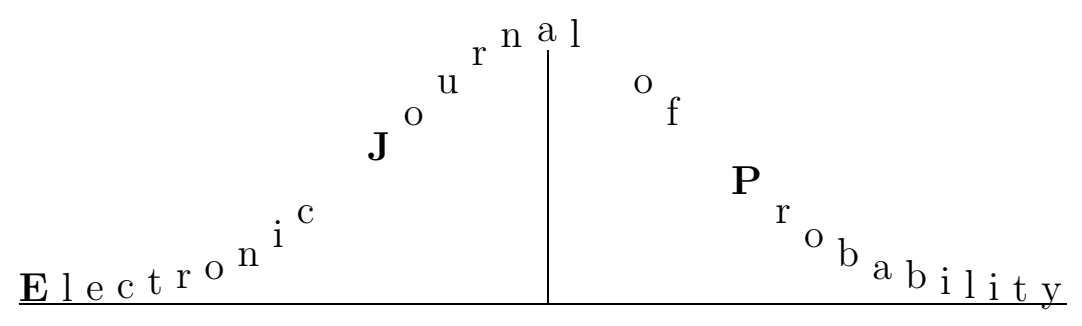

Vol. 10 (2005), Paper no. 44, pages 1442-1467.

Journal URL

http://www.math.washington.edu/ ejpecp/

\title{
Estimates of Random Walk Exit Probabilities and Application to Loop-Erased Random Walk
}

\author{
Michael J. Kozdron ${ }^{1}$ \\ Department of Mathematics \& Statistics, College West 307.14 \\ University of Regina, Regina, SK S4S 0A2 Canada \\ kozdron@math. uregina.ca \\ and \\ Gregory F. Lawler ${ }^{2}$ \\ Department of Mathematics, 310 Malott Hall \\ Cornell University, Ithaca, NY 14853-4201 USA \\ lawler@math. cornell.edu
}

\begin{abstract}
We prove an estimate for the probability that a simple random walk in a simply connected subset $A \subset \mathbb{Z}^{2}$ starting on the boundary exits $A$ at another specified boundary point. The estimates are uniform over all domains of a given inradius. We apply these estimates to prove a conjecture of S. Fomin [4] in 2001 concerning a relationship between crossing probabilities of loop-erased random walk and Brownian motion.
\end{abstract}

2000 Mathematics Subject Classification. 60F99, 60G50, 60J45, 60J65

Key words and phrases. Brownian motion, loop-erased random walk, Green's function estimates, excursion Poisson kernel, Fomin's identity, strong approximation.

Submitted to EJP on March 20, 2005. Final version accepted on November 22, 2005.

\footnotetext{
${ }^{1}$ Research supported in part by the Natural Sciences and Engineering Research Council of Canada.

${ }^{2}$ Research supported by the National Science Foundation.
} 


\section{Introduction}

In the last few years a number of results have been proved about scaling limits of twodimensional lattice systems in statistical mechanics. Site percolation on the triangular lattice [16], loop-erased random walk [14, 17], uniform spanning trees [14], and the harmonic explorer [15] have all been shown to have limits that can be described using the SchrammLoewner evolution. In the last three cases, the proofs use a version of the well-known fact that simple random walk has a scaling limit of Brownian motion, which is conformally invariant in two dimensions. What is needed is a strong version of this result which holds uniformly over a wide class of domains where the errors do not depend on the smoothness of the boundary. In this paper, we present another result of this type. It differs from the lemmas in [14] in two ways: we give explicit error bounds that show that the error decays as a power of the "inradius" of the domain, and the continuous domain that we compare to the discrete domain is slightly different.

We give an application of our result to loop-erased walk by proving a conjecture of S. Fomin [4] and giving a quick derivation of a crossing exponent first proved by R. Kenyon [6]. Fomin showed that a certain crossing probability for loop-erased walk can be given in terms of a determinant of hitting probabilities for simple random walk. Our estimate shows that this determinant approaches a corresponding determinant for Brownian motion. We then estimate the determinant for Brownian motion to derive the crossing exponent.

We will start by discussing the main results, leaving some of the precise definitions until Section 2. The only Euclidean dimension that will concern us is $d=2$; consequently, we associate $\mathbb{C} \cong \mathbb{R}^{2}$ in the natural way. Points in the complex plane will be denoted by any of $w, x, y$, or $z$. A domain $D \subset \mathbb{C}$ is an open and connected set.

Throughout this paper, $B_{t}, t \geq 0$, will denote a standard complex Brownian motion, and $S_{n}, n=0,1, \ldots$, will denote two-dimensional simple random walk, both started at the origin unless otherwise noted. We write $B[0, t]=\left\{z \in \mathbb{C}: B_{s}=z\right.$ for some $\left.0 \leq s \leq t\right\}$, and $S[0, n]=\left[S_{0}, S_{1}, \ldots, S_{n}\right]$ for the set of lattice points visited by the random walk. We will generally use $T$ for stopping times for Brownian motion, and $\tau$ for stopping times for random walk. We write $\mathbb{E}^{x}$ and $\mathbb{P}^{x}$ for expectations and probabilities, respectively, assuming $B_{0}=x$ or $S_{0}=x$, as appropriate.

\subsection{Main results}

Let $\mathcal{A}^{n}$ denote the collection of simply connected subsets $A$ of $\mathbb{Z}^{2}$ such that $n \leq \operatorname{inrad}(A) \leq$ $2 n$, i.e., such that

$$
n \leq \sup \left\{|z|: z \in \mathbb{Z}^{2} \backslash A\right\} \leq 2 n .
$$

Associated to $A$ is a simply connected domain $\tilde{A} \subset \mathbb{C}$ which is obtained by identifying each lattice point in $A$ with the square of side one centred at that point. By the Riemann mapping theorem, there is a unique conformal transformation $f_{A}$ of $\tilde{A}$ onto the unit disk with $f_{A}(0)=0, f_{A}^{\prime}(0)>0$. We let $\theta_{A}(x)=\arg \left(f_{A}(x)\right)$. We can extend $\theta_{A}$ to $\partial A$ in a natural way.

If $x \in A, y \in \partial A$, let $h_{A}(x, y)$ be the probability that a simple random walk starting at $x$ leaves $A$ at $y$. If $x, y \in \partial A$, let $h_{\partial A}(x, y)$ be the probability that a simple random walk starting at $x$ takes its first step into $A$ and then leaves $A$ at $y$. 
Theorem 1.1. If $A \in \mathcal{A}^{n}$, then

$$
h_{\partial A}(x, y)=\frac{(\pi / 2) h_{A}(0, x) h_{A}(0, y)}{1-\cos \left(\theta_{A}(x)-\theta_{A}(y)\right)}\left[1+O\left(\frac{\log n}{n^{1 / 16}\left|\theta_{A}(x)-\theta_{A}(y)\right|}\right)\right],
$$

provided that $\left|\theta_{A}(x)-\theta_{A}(y)\right| \geq n^{-1 / 16} \log ^{2} n$.

In this theorem, and throughout this paper, we will use $O(\cdot)$ for uniform error terms that depend only on $n$. For example, the statement above is shorthand for the following: there is a constant $c<\infty$ such that for all $A \in \mathcal{A}^{n}$ and all $x, y \in \partial A$ with $\left|\theta_{A}(x)-\theta_{A}(y)\right| \geq$ $n^{-1 / 16} \log ^{2} n$,

$$
\left|h_{\partial A}(x, y)-\frac{(\pi / 2) h_{A}(0, x) h_{A}(0, y)}{1-\cos \left(\theta_{A}(x)-\theta_{A}(y)\right)}\right| \leq c \frac{(\pi / 2) h_{A}(0, x) h_{A}(0, y)}{1-\cos \left(\theta_{A}(x)-\theta_{A}(y)\right)} \frac{\log n}{n^{1 / 16}\left|\theta_{A}(x)-\theta_{A}(y)\right|} .
$$

When discussing $k$-fold determinants, we will have error terms that depend also on a positive integer $k$; we will write these as $O_{k}(\cdot)$.

We do not believe the error term $O\left(n^{-1 / 16} \log n\right)$ is optimal, and we probably could have improved it slightly in this paper. However, our methods are not strong enough to give the optimal error term. The importance of this result is that the error is bounded uniformly over all simply connected domains and that the error is in terms of a power of $n$. For domains with "smooth" boundaries, one can definitely improve the power of $n$.

To help understand this estimate, one should consider $h_{\partial A}(x, y)$ as having a "local" and a "global" part. The local part, which is very dependent on the structure of $A$ near $x$ and $y$, is represented by the $h_{A}(0, x) h_{A}(0, y)$ term. The global part, which is $\left[1-\cos \left(\theta_{A}(x)-\theta_{A}(y)\right)\right]^{-1}$, is the conformal invariant and depends only on the image of the points under the conformal transformation of $\tilde{A}$ onto the unit disk. In contrast to the discrete case, Example 2.14 shows that the Brownian version of this result is exact.

As part of the proof, we also derive a uniform estimate for $G_{A}(x)$, the expected number of visits to $x$ before leaving $A$ of a simple random walk starting at 0 . Let $a$ denote the potential kernel for two-dimensional simple random walk. It is known that there is a $k_{0}$ such that

$$
a(x)=\frac{2}{\pi} \log |x|+k_{0}+O\left(|x|^{-2}\right), \quad x \rightarrow \infty .
$$

Theorem 1.2. If $A \in \mathcal{A}^{n}$, then

$$
G_{A}(0)=-\frac{2}{\pi} \log f_{A}^{\prime}(0)+k_{0}+O\left(n^{-1 / 3} \log n\right) .
$$

Furthermore, if $x \neq 0$, then

$$
G_{A}(x)=\frac{2}{\pi} g_{A}(x)+k_{x}+O\left(n^{-1 / 3} \log n\right) .
$$

where $g_{A}(x)=g_{A}(0, x)=-\log \left|f_{A}(x)\right|$ is the Green's function for Brownian motion in $\tilde{A}$ and

$$
k_{x}=k_{0}+\frac{2}{\pi} \log |x|-a(x) .
$$




\subsection{Fomin's identity for loop-erased walk}

We briefly review the definition of the loop-erased random walk; see [11, Chapter 7] and [12] for more details. Since simple random walk in $\mathbb{Z}^{2}$ is recurrent, it is not possible to construct loop-erased random walk by erasing loops from an infinite walk. However, the following loop-erasing procedure makes perfect sense since it assigns to each finite simple random walk path a self-avoiding walk. Let $S=S[0, m]=\left[S_{0}, S_{1}, \ldots, S_{m}\right]$ be a simple random walk path of length $m$. We construct $\mathcal{L}(S)$, the loop-erased part of $S$, recursively as follows. If $S$ is already self-avoiding, set $\mathcal{L}(S)=S$. Otherwise, let $s_{0}=\max \left\{j: S_{j}=S_{0}\right\}$, and for $i>0$, let $s_{i}=\max \left\{j: S_{j}=S_{s_{i-1}+1}\right\}$. If we let $n=\min \left\{i: s_{i}=m\right\}$, then $\mathcal{L}(S)=\left[S_{s_{0}}, S_{s_{1}}, \ldots, S_{s_{n}}\right]$.

Suppose that $A \in \mathcal{A}^{n}$ and $x^{1}, \ldots, x^{k}, y^{k}, \ldots, y^{1}$ are distinct points in $\partial A$, ordered counterclockwise. For $i=1, \ldots, k$, let $\mathcal{L}^{i}=\mathcal{L}\left(S^{i}\right)$ be the loop erasure of the path $\left[S_{0}^{i}=\right.$ $\left.x^{i}, S_{1}^{i}, \ldots, S_{\tau_{A}^{i}}^{i}\right]$, and let $\mathcal{C}=\mathcal{C}\left(x^{1}, \ldots, x^{k}, y^{k}, \ldots, y^{1} ; A\right)$ be the event that both

$$
S_{\tau_{A}^{i}}^{i}=y^{i}, \quad i=1, \ldots, k,
$$

and

$$
S^{i}\left[0, \tau_{A}^{i}\right] \cap\left(\mathcal{L}^{1} \cup \cdots \cup \mathcal{L}^{i-1}\right)=\emptyset, \quad i=2, \ldots, k .
$$

The following theorem was proved in [4] which relates a determinant of simple random walk probabilities to a "crossing probability" for loop-erased random walk.

Theorem 1.3 (Fomin). If $\mathcal{C}$ is the event defined above, and

$$
\mathbf{h}_{\partial A}(\mathbf{x}, \mathbf{y})=\left[\begin{array}{ccc}
h_{\partial A}\left(x^{1}, y^{1}\right) & \cdots & h_{\partial A}\left(x^{1}, y^{k}\right) \\
\vdots & \ddots & \vdots \\
h_{\partial A}\left(x^{k}, y^{1}\right) & \cdots & h_{\partial A}\left(x^{k}, y^{k}\right)
\end{array}\right],
$$

where $\mathbf{x}=\left(x^{1}, \ldots, x^{k}\right), \mathbf{y}=\left(y^{1}, \ldots, y^{k}\right)$, then $\mathbb{P}(\mathcal{C})=\operatorname{det} \mathbf{h}_{\partial A}(\mathbf{x}, \mathbf{y})$.

This is a special case of an identity that Fomin established for general discrete stationary Markov processes. In his paper, he made the following conjecture.

In order for the statement of Theorem 1.3 to make sense, the Markov process under consideration does not have to be discrete.... The proofs can be obtained by passing to a limit in the discrete approximation. The same limiting procedure can be used to justify the well-definedness of the quantities involved; notice that in order to define a continuous analogue of Theorem 1.3, we do not need the notion of loop-erased Brownian motion. Instead, we discretize the model, compute the probability, and then pass to the limit. One can further extend these results to densities of the corresponding hitting distributions. Technical details are omitted.

With Theorem 1.1, we have taken care of the "technical details" in the case of simply connected planar domains. Note that

$$
\operatorname{det}\left[\frac{h_{\partial A}\left(x^{j}, y^{l}\right)}{h_{\partial A}\left(x^{j}, y^{j}\right)}\right]_{1 \leq j, l \leq k}=\frac{\operatorname{det} \mathbf{h}_{\partial A}(\mathbf{x}, \mathbf{y})}{\prod_{j=1}^{k} h_{\partial A}\left(x^{j}, y^{j}\right)}
$$


represents the conditional probability that (3) holds given (2) holds. Suppose $D$ is a smooth Jordan domain, and that $x^{1}, \ldots, x^{k}, y^{k}, \ldots, y^{1}$ are distinct points on $\partial D$ ordered counterclockwise. The "Brownian motion" analogue of the determinant (4) is

$$
\Lambda_{D}\left(x^{1}, \ldots, x^{k}, y^{k}, \ldots, y^{1}\right)=\operatorname{det}\left[\frac{H_{\partial D}\left(x^{j}, y^{l}\right)}{H_{\partial D}\left(x^{j}, y^{j}\right)}\right]_{1 \leq j, l \leq k}=\frac{\operatorname{det} \mathbf{H}_{\partial D}(\mathbf{x}, \mathbf{y})}{\prod_{j=1}^{k} H_{\partial D}\left(x^{j}, y^{j}\right)}
$$

where $H_{\partial D}(z, w)$ denotes the excursion Poisson kernel. In the case $D=\mathbb{D}$, if $z=e^{i \theta}, w=e^{i \theta^{\prime}}$, then

$$
H_{\partial \mathbb{D}}(z, w)=\frac{1}{\pi} \frac{1}{|w-z|^{2}}=\frac{1}{2 \pi} \frac{1}{1-\cos \left(\theta^{\prime}-\theta\right)} .
$$

Conformal covariance of the excursion Poisson kernel shows that

$$
\begin{aligned}
\Lambda_{D}\left(x^{1}, \ldots, x^{k}, y^{k}, \ldots, y^{1}\right) & =\Lambda_{\mathbb{D}}\left(f\left(x^{1}\right), \ldots, f\left(x^{k}\right), f\left(y^{k}\right), \ldots, f\left(y^{1}\right)\right) \\
& =\operatorname{det}\left[\frac{1-\cos \left(\theta_{D}\left(x^{j}\right)-\theta_{D}\left(y^{j}\right)\right)}{1-\cos \left(\theta_{D}\left(x^{j}\right)-\theta_{D}\left(y^{l}\right)\right)}\right]_{1 \leq j, l \leq k},
\end{aligned}
$$

where $f$ is a conformal transformation of $D$ onto $\mathbb{D}$ and $\theta_{D}(z)=\arg (f(z))$.

Corollary 1.4. Suppose $A \in \mathcal{A}^{n}$ and $x^{1}, \ldots, x^{k}, y^{k}, \ldots, y^{1}$ are distinct points in $\partial A$ ordered counterclockwise. Let

$$
m=\min \left\{\left|\theta_{A}\left(x^{1}\right)-\theta_{A}\left(y^{1}\right)\right|,\left|\theta_{A}\left(x^{k}\right)-\theta_{A}\left(y^{k}\right)\right|\right\} .
$$

If $m \geq n^{-1 / 16} \log ^{2} n$, then

$$
\operatorname{det}\left[\frac{h_{\partial A}\left(x^{j}, y^{l}\right)}{h_{\partial A}\left(x^{j}, y^{j}\right)}\right]_{1 \leq j, l \leq k}=\operatorname{det}\left[\frac{1-\cos \left(\theta_{A}\left(x^{j}\right)-\theta_{A}\left(y^{j}\right)\right)}{1-\cos \left(\theta_{A}\left(x^{j}\right)-\theta_{A}\left(y^{l}\right)\right)}\right]_{1 \leq j, l \leq k}+O_{k}\left(\frac{\log n}{n^{1 / 16} m^{2 k+1}}\right) .
$$

Proof. Theorem 1.1 gives

$$
\operatorname{det}\left[\frac{h_{\partial A}\left(x^{j}, y^{l}\right)}{h_{\partial A}\left(x^{j}, y^{j}\right)}\right]_{1 \leq j, l \leq k}=\operatorname{det}\left[\frac{1-\cos \left(\theta_{A}\left(x^{j}\right)-\theta_{A}\left(y^{j}\right)\right)}{1-\cos \left(\theta_{A}\left(x^{j}\right)-\theta_{A}\left(y^{l}\right)\right)}\left[1+O\left(\frac{\log n}{m n^{1 / 16}}\right)\right]\right]_{1 \leq j, l \leq k} .
$$

But, if $\left|\delta_{j, l}\right| \leq \varepsilon$, multilinearity of the determinant and the estimate $\operatorname{det}\left[b_{j, l}\right] \leq k^{k / 2}\left[\sup \left|b_{j, l}\right|\right]^{k}$ shows that

$$
\left|\operatorname{det}\left[b_{j, l}\left(1+\delta_{j, l}\right)\right]-\operatorname{det}\left[b_{j, l}\right]\right| \leq\left[(1+\varepsilon)^{k}-1\right] k^{k / 2}\left[\sup \left|b_{j, l}\right|\right]^{k} .
$$

Using the corollary, we know that we can approximate the determinant for random walks, and hence the probability of the crossing event $\mathcal{C}$, in terms of the corresponding quantity for Brownian motion, at least for simply connected domains. We will consider the asymptotics of $\Lambda_{D}\left(x^{1}, \ldots, x^{k}, y^{k}, \ldots, y^{1}\right)$ when $x^{1}, \ldots, x^{k}$ get close and $y^{1}, \ldots, y^{k}$ get close. Since this quantity is a conformal invariant, we may assume that $D=\mathcal{R}_{L}$, where

$$
\mathcal{R}_{L}=\{z: 0<\operatorname{Re}(z)<L, 0<\operatorname{Im}(z)<\pi\},
$$

and $x^{j}=i q_{j}, y^{j}=L+i q_{j}^{\prime}$, where $0<q_{k}<\cdots<q_{1}<\pi$ and $0<q_{k}^{\prime}<\cdots<q_{1}^{\prime}<\pi$. 
Proposition 1.5. As $L \rightarrow \infty$,

$$
\begin{aligned}
& \Lambda_{\mathcal{R}_{L}}\left(i q_{1}, \ldots, i q_{k}, L+i q_{k}^{\prime}, \ldots, L+i q_{1}^{\prime}\right) \\
& =k ! \frac{\operatorname{det}\left[\sin \left(l q_{j}\right)\right]_{1 \leq j, l \leq k} \operatorname{det}\left[\sin \left(l q_{j}^{\prime}\right)\right]_{1 \leq j, l \leq k}}{k} e^{-k(k-1) L / 2}+O_{k}\left(e^{-k(k+1) L / 2}\right) . \\
& \prod_{j=1}^{k} \sin \left(q_{j}\right) \sin \left(q_{j}^{\prime}\right)
\end{aligned}
$$

This crossing exponent $k(k-1) / 2$ was first proved by Kenyon [6] for loop-erased walk. We describe this result in the framework of Brownian excursion measure.

\subsection{Outline of the paper}

In the first five subsections of Section 2, we review facts about random walk, Brownian motion, and conformal mapping that we will need. In the remaining subsections, we review Brownian excursion measure, define the analogue of the Fomin determinant for excursion measure, and then derive Proposition 1.5. In Section 3, we begin with a brief review of strong approximation, before proving Theorem 1.2 in the second subsection. The final two subsections contain the proof of the other main result, Theorem 1.1.

\section{Background, notation, and preliminary results}

In this section we review some basic material that will be needed in subsequent parts and standardize our notation. Almost all of the complex analysis is well-known, and may be found in a variety of sources; we prove several elementary results, but often refer the reader to the literature for details. The material on the excursion Poisson kernel is not difficult, but these results are not widespread.

\subsection{Simply connected subsets of $\mathbb{C}$ and $\mathbb{Z}^{2}$}

We will use $D$ to denote domains, i.e., open connected subsets of $\mathbb{C}$. We write $\mathbb{D}=\{z \in \mathbb{C}$ : $|z|<1\}$ to denote the open unit disk, and $\mathbb{H}=\{z \in \mathbb{C}: \operatorname{Im}(z)>0\}$ to denote the upper half plane. An analytic, univalent (i.e, one-to-one) function ${ }^{3}$ is called a conformal mapping. We say that $f: D \rightarrow D^{\prime}$ is a conformal transformation if $f$ is a conformal mapping that is onto $D^{\prime}$. It follows that $f^{\prime}(z) \neq 0$ for $z \in D$, and $f^{-1}: D^{\prime} \rightarrow D$ is also a conformal transformation. We write $\mathcal{S}$ to denote the set of functions $f$ which are analytic and univalent in $\mathbb{D}$ satisfying the normalizing conditions $f(0)=0$ and $f^{\prime}(0)=1$.

If $D \subset \mathbb{C}$ with $0 \in D$, we define the radius (with respect to the origin) of $D$ to be $\operatorname{rad}(D)=\sup \{|z|: z \in \partial D\}$, and the inradius (with respect to the origin) of $D$ to be $\operatorname{inrad}(D)=\operatorname{dist}(0, \partial D)=\inf \{|z|: z \in \partial D\}$. The diameter of $D$ is given by $\operatorname{diam}(D)=$ $\sup \{|x-y|: x, y \in D\}$. If $D \subset \mathbb{C}$, then we say that a bounded $D$ is a Jordan domain if $\partial D$ is a Jordan curve (i.e., homeomorphic to a circle). A Jordan domain is nice if the

\footnotetext{
${ }^{3}$ For an analytic function $f, f^{\prime}\left(z_{0}\right) \neq 0$ if and only if $f$ is locally univalent at $z_{0}$. However, we will not be concerned with local univalence.
} 
Jordan curve $\partial D$ can be expressed as a finite union of analytic curves. Note that Jordan domains are simply connected. For each $r>0$, let $\mathcal{D}^{r}$ be the set of nice Jordan domains containing the origin of inradius $r$, and write $\mathcal{D}=\bigcup_{r>0} \mathcal{D}^{r}$. We also define $\mathcal{D}^{*}$ to the be set of Jordan domains containing the origin, and note that $\mathbb{D} \in \mathcal{D} \subset \mathcal{D}^{*}$. If $D, D^{\prime} \in \mathcal{D}^{*}$, let $\mathcal{T}\left(D, D^{\prime}\right)$ be the set of all $f: D \rightarrow D^{\prime}$ that are conformal transformations of $D$ onto $D^{\prime}$. The Riemann mapping theorem implies that $\mathcal{T}\left(D, D^{\prime}\right) \neq \emptyset$, and since $\partial D, \partial D^{\prime}$ are Jordan, the Carathéodory extension theorem tells us that $f \in \mathcal{T}\left(D, D^{\prime}\right)$ can be extended to a homeomorphism of $\bar{D}$ onto $\overline{D^{\prime}}$. We will use this fact repeatedly throughout, without explicit mention of it. For statements and details on these two theorems, consult [3, §1.5].

A subset $A \subset \mathbb{Z}^{2}$ is connected if every two points in $A$ can be connected by a nearest neighbour path staying in $A$. We say that a finite subset $A$ is simply connected if both $A$ and $\mathbb{Z}^{2} \backslash A$ are connected. There are three standard ways to define the "boundary" of a proper subset $A$ of $\mathbb{Z}^{2}$ :

- (outer) boundary: $\partial A=\left\{y \in \mathbb{Z}^{2} \backslash A:|y-x|=1\right.$ for some $\left.x \in A\right\}$;

- inner boundary: $\partial_{i} A=\partial\left(\mathbb{Z}^{2} \backslash A\right)=\left\{x \in A:|y-x|=1\right.$ for some $\left.y \in \mathbb{Z}^{2} \backslash A\right\}$;

- edge boundary: $\partial_{e} A=\left\{(x, y): x \in A, y \in \mathbb{Z}^{2} \backslash A,|x-y|=1\right\}$.

To each finite, connected $A \subset \mathbb{Z}^{2}$ we associate a domain $\tilde{A} \subset \mathbb{C}$ in the following way. For each edge $(x, y) \in \partial_{e} A$, considered as a line segment of length one, let $\ell_{x, y}$ be the perpendicular line segment of length one intersecting $(x, y)$ in the midpoint. Let $\partial \tilde{A}$ denote

the union of the line segments $\ell_{x, y}$, and let $\tilde{A}$ denote the domain with boundary $\partial \tilde{A}$ containing A. Observe that

$$
\tilde{A} \cup \partial \tilde{A}=\bigcup_{x \in A} \mathcal{S}_{x} \text { where } \mathcal{S}_{x}=x+([-1 / 2,1 / 2] \times[-1 / 2,1 / 2]) .
$$

That is, $\mathcal{S}_{x}$ is the closed square of side length one centred at $x$ whose sides are parallel to the coordinate axes. Also, note that $\tilde{A}$ is simply connected if and only if $A$ is a simply connected subset of $\mathbb{Z}^{2}$. We refer to $\tilde{A}$ as the "union of squares" domain associated to $A$.

Let $\mathcal{A}$ denote the set of all finite simply connected subsets of $\mathbb{Z}^{2}$ containing the origin. If $A \in \mathcal{A}$, let $\operatorname{inrad}(A)=\min \left\{|z|: z \in \mathbb{Z}^{2} \backslash A\right\}$ and $\operatorname{rad}(A)=\max \{|z|: z \in A\}$ denote the inradius and radius (with respect to the origin), respectively, of $A$, and define $\mathcal{A}^{n}$ to be the set of $A \in \mathcal{A}$ with $n \leq \operatorname{inrad}(A) \leq 2 n$; thus $\mathcal{A}=\bigcup_{n \geq 0} \mathcal{A}^{n}$. Note that if $A \in \mathcal{A}$ and $0 \neq x \in \partial_{i} A$, then the connected component of $A \backslash\{x\}$ containing the origin is simply connected. (This is not true if we do not assume $x \in \partial_{i} A$.) Similarly, by induction, if $A \in \mathcal{A}$, $0 \neq x_{1} \in \partial_{i} A$, and $\left[x_{1}, x_{2}, \ldots, x_{j}\right]$ is a nearest neighbour path in $A \backslash\{0\}$, then the connected component of $A \backslash\left\{x_{1}, \ldots, x_{j}\right\}$ containing the origin is simply connected.

Finally, if $A \in \mathcal{A}$ with associated domain $\tilde{A} \subset \mathbb{C}$, then we write $f_{A}=f_{\tilde{A}}$ for the conformal transformation of $\tilde{A}$ onto the unit disk $\mathbb{D}$ with $f_{A}(0)=0, f_{A}^{\prime}(0)>0$.

\subsection{Green's functions on $\mathbb{C}$}

If $D$ is a domain whose boundary includes a curve, let $g_{D}(x, y)$ denote the Green's function for Brownian motion. If $x \in D$, we can define $g_{D}(x, \cdot)$ as the unique harmonic function 
on $D \backslash\{x\}$, vanishing on $\partial D$ (in the sense that $g_{D}(x, y) \rightarrow 0$ as $y \rightarrow y_{0}$ for every regular $\left.y_{0} \in \partial D\right)$, with

$$
g_{D}(x, y)=-\log |x-y|+O(1) \text { as }|x-y| \rightarrow 0 .
$$

In the case $D=\mathbb{D}$, we have

$$
g_{\mathbb{D}}(x, y)=\log |\bar{y} x-1|-\log |y-x| .
$$

Note that $g_{\mathbb{D}}(0, x)=-\log |x|$, and $g_{\mathbb{D}}(x, y)=g_{\mathbb{D}}(y, x)$. The Green's function is a well-known example of a conformal invariant (see, e.g., $[3, \S 1.8]$ ).

Proposition 2.1 (Conformal Invariance of the Green's Function). Suppose that $f$ : $D \rightarrow D^{\prime}$ is a conformal transformation. If $x, y \in D$, then $g_{D}(x, y)=g_{D^{\prime}}(f(x), f(y))$.

Hence, to determine $g_{D}$ for arbitrary $D \in \mathcal{D}^{*}$, it is enough to find $f_{D} \in \mathcal{T}(D, \mathbb{D})$. On the other hand, suppose $D \subset \mathbb{C}$ is a simply connected domain containing the origin with Green's function $g_{D}(z, w)$. Then we can write the unique conformal transformation of $D$ onto $\mathbb{D}$ with $f_{D}(0)=0, f_{D}^{\prime}(0)>0$ as

$$
f_{D}(x)=\exp \left\{-g_{D}(x)+i \theta_{D}(x)\right\},
$$

where $g_{D}(x)=g_{D}(0, x)$ and $-g_{D}+i \theta_{D}$ is analytic in $D \backslash\{0\}$.

An equivalent formulation of the Green's function for $D \in \mathcal{D}^{*}$ can be given in terms of Brownian motion. Using (8) we have that $g_{D}(x, y)=\mathbb{E}^{x}\left[\log \left|B_{T_{D}}-y\right|\right]-\log |x-y|$ for distinct points $x, y \in D$ where $T_{D}=\inf \left\{t: B_{t} \notin D\right\}$. In particular, if $0 \in D$, then

$$
g_{D}(x)=\mathbb{E}^{x}\left[\log \left|B_{T_{D}}\right|\right]-\log |x| \text { for } x \in D .
$$

Additional details may be found in [13, Chapter 2].

If $A \in \mathcal{A}$, and if we let $g_{A}(x, y)=g_{\tilde{A}}(x, y)$ be the Green's function (for Brownian motion) in $\tilde{A}$, then by Proposition 2.1 and (9) we have that

$$
g_{A}(x, y)=g_{\mathbb{D}}\left(f_{A}(x), f_{A}(y)\right)=\log \left|\frac{\overline{f_{A}(y)} f_{A}(x)-1}{f_{A}(y)-f_{A}(x)}\right| .
$$

If we write $\theta_{A}=\theta_{\tilde{A}}$, then (10) implies that

$$
f_{A}(x)=\exp \left\{-g_{A}(x)+i \theta_{A}(x)\right\} .
$$

\subsection{Green's functions on $\mathbb{Z}^{2}$}

Suppose that $S_{n}$ is a simple random walk on $\mathbb{Z}^{2}$ and $A$ is a proper subset of $\mathbb{Z}^{2}$. If $\tau_{A}=$ $\min \left\{j \geq 0: S_{j} \notin A\right\}$, then we let

$$
G_{A}(x, y)=\mathbb{E}^{x}\left[\sum_{j=0}^{\tau_{A}-1} \cdot\left\{S_{j}=y\right\}\right]=\sum_{j=0}^{\infty} \mathbb{P}^{x}\left\{S_{j}=y, \tau_{A}>j\right\}
$$


denote the Green's function for random walk on $A$. Set $G_{A}(x)=G_{A}(x, 0)=G_{A}(0, x)$. In analogy with the Brownian motion case (see [11, Proposition 1.6.3]), we have

$$
G_{A}(x)=\mathbb{E}^{x}\left[a\left(S_{\tau_{A}}\right)\right]-a(x) \text { for } x \in A
$$

where $a$ is the potential kernel for simple random walk defined by

$$
a(x)=\sum_{j=0}^{\infty}\left[\mathbb{P}^{0}\left\{S_{j}=0\right\}-\mathbb{P}^{x}\left\{S_{j}=0\right\}\right] .
$$

It is also known [11, Theorem 1.6.2] that as $|x| \rightarrow \infty$,

$$
a(x)=\frac{2}{\pi} \log |x|+k_{0}+o\left(|x|^{-3 / 2}\right)
$$

where $k_{0}=(2 \varsigma+3 \ln 2) / \pi$ and $\varsigma$ is Euler's constant. The error above will suffice for our purposes, even though stronger results are known. The asymptotic expansion of $a(x)$ given in [5] shows that the error is $O\left(|x|^{-2}\right)$.

\subsection{Consequences of the Koebe theorems}

We now recall some standard results from the study of univalent functions. Proofs may be found in [3, Theorems 2.3, 2.4, 2.5, 2.6].

Theorem 2.2 (Koebe One-Quarter Theorem). If $f$ is a conformal mapping of the unit disk with $f(0)=0$, then the image of $f$ contains the open disk of radius $\left|f^{\prime}(0)\right| / 4$ about the origin.

Theorem 2.3 (Koebe Growth and Distortion Theorem). If $f \in \mathcal{S}$ and $z \in \mathbb{D}$, then

$$
\begin{gathered}
\left|\frac{z f^{\prime \prime}(z)}{f^{\prime}(z)}-\frac{2|z|^{2}}{1-|z|^{2}}\right| \leq \frac{4|z|}{1-|z|^{2}}, \quad \frac{|z|}{(1+|z|)^{2}} \leq|f(z)| \leq \frac{|z|}{(1-|z|)^{2}} \\
\text { and } \frac{1-|z|}{(1+|z|)^{3}} \leq\left|f^{\prime}(z)\right| \leq \frac{1+|z|}{(1-|z|)^{3}} .
\end{gathered}
$$

A number of useful consequences may now be deduced.

Corollary 2.4. For each $0<r<1$, there is a constant $c_{r}$ such that if $f \in \mathcal{S}$ and $|z| \leq r$, then $|f(z)-z| \leq c_{r}|z|^{2}$.

Proof. If we combine the first estimate in Theorem 2.3 with the estimate of $\left|f^{\prime}(z)\right|$ in the third statement of that theorem, then we can obtain a uniform bound on $\left|f^{\prime \prime}(z)\right|$ over all $f \in \mathcal{S}$ and $|z| \leq r$.

Recall that $f_{A}=f_{\tilde{A}} \in \mathcal{T}(\tilde{A}, \mathbb{D})$ is the unique conformal transformation of $\tilde{A}$ onto $\mathbb{D}$ with $f_{A}(0)=0, f_{A}^{\prime}(0)>0$.

Corollary 2.5. If $A \in \mathcal{A}^{n}$, then $-\log f_{A}^{\prime}(0)=\log n+O(1)$. 
Proof. Using the Koebe one-quarter theorem and the Schwarz lemma, we see that if $F$ : $\mathbb{D} \rightarrow D$ is a conformal transformation with $F(0)=0$, then

$$
\left|F^{\prime}(0)\right| / 4 \leq \operatorname{inrad}(D) \leq\left|F^{\prime}(0)\right|
$$

By definition, since $A \in \mathcal{A}^{n}$, we have that $n-1 \leq \operatorname{inrad}(\tilde{A}) \leq 2 n+1$. Therefore, if $F_{A}=f_{A}^{-1}$, then

$$
n-1 \leq F_{A}^{\prime}(0) \leq 4(2 n+1) .
$$

Along with Corollary 2.4, the growth and distortion theorem yields the following.

Corollary 2.6. If $A \in \mathcal{A}^{n}$ and $|x| \leq n / 16$, then $f_{A}(x)=x f_{A}^{\prime}(0)+|x|^{2} O\left(n^{-2}\right)$, and

$$
g_{A}(x)+\log |x|=-\log f_{A}^{\prime}(0)+|x| O\left(n^{-1}\right) .
$$

Proof. For $z \in \mathbb{D}$, let $F_{A}(z)=f_{A}(n z) /\left(n f_{A}^{\prime}(0)\right)$. Then $F_{A} \in \mathcal{S}$, so Corollary 2.4 with $r=1 / 16$ gives $\left|F_{A}(z)-z\right| \leq C|z|^{2}$. Thus, if $z=x / n,\left|f_{A}(x)-x f_{A}^{\prime}(0)\right| \leq C f_{A}^{\prime}(0)|x|^{2} n^{-1}$. By the

previous corollary, $f_{A}^{\prime}(0)=O\left(n^{-1}\right)$, so the first assertion follows. The second result follows from $\left|f_{A}(x)\right|=\exp \left\{-g_{A}(x)\right\}$.

We remark that this corollary implies $\lim _{|x| \rightarrow 0}\left(g_{A}(x)+\log |x|\right)=-\log f_{A}^{\prime}(0)$ which shows the size of the error term in (8).

\subsection{Beurling estimates and related results}

Throughout this subsection, suppose that $A \in \mathcal{A}^{n}$ with associated "union of squares" domain $\tilde{A} \subset \mathbb{C}$, and write $T_{A}=T_{\tilde{A}}=\inf \left\{t: B_{t} \notin \tilde{A}\right\}$. The following may be derived from the Beurling projection theorem [2, Theorem (V.4.1)].

Theorem 2.7. There is a constant $c<\infty$ such that if $\gamma:[a, b] \rightarrow \mathbb{C}$ is a curve with $|\gamma(a)|=r,|\gamma(b)|=R, 0<r<R<\infty, \gamma(a, b) \subset \mathbb{D}_{R}=\{z \in \mathbb{C}:|z|<R\}$, and $|x| \leq r$, then

$$
\mathbb{P}^{x}\left\{B\left[0, T_{\mathbb{D}_{R}}\right] \cap \gamma[a, b]=\emptyset\right\} \leq c(r / R)^{1 / 2}
$$

Corollary 2.8 (Beurling Estimate). There is a constant $c<\infty$ such that if $x \in \tilde{A}$, then for all $r>0$,

$$
\mathbb{P}^{x}\left\{\left|B_{T_{A}}-x\right|>r \operatorname{dist}(x, \partial \tilde{A})\right\} \leq c r^{-1 / 2}
$$

Proof. Without loss of generality, we may assume by Brownian scaling that $x=0$ and $d=\operatorname{inrad}(\tilde{A}) \in[1 / 2,1]$. If $\operatorname{rad}(\tilde{A}) \leq r$, then this estimate is trivial. If not, then there is a curve in $\partial \tilde{A}$ from the circle of radius $d$ to the circle of radius $r$, and the Beurling estimate (19) follows from (18).

In particular, if $|x|>n / 2$, the probability starting at $x$ of reaching $\mathbb{D}_{n / 2}$ before leaving $\tilde{A}$ i s bounded above by $c n^{-1 / 2} \operatorname{dist}(x, \partial \tilde{A})^{1 / 2}$. It follows easily from the Koebe one-quarter theorem that $g_{A}(x) \leq c$ for $|x| \geq n / 4$; hence we get

$$
g_{A}(x) \leq c n^{-1 / 2} \operatorname{dist}(x, \partial \tilde{A})^{1 / 2}, \quad A \in \mathcal{A}^{n}, \quad|x| \geq n / 4 .
$$


Recall from (13) that $f_{A}(x)=\exp \left\{-g_{A}(x)+i \theta_{A}(x)\right\}$ for $x \in \tilde{A}$. Hence, if $x \in \partial_{i} A$, then $g_{A}(x) \leq c n^{-1 / 2}$, so that $f_{A}(x)=\exp \left\{i \theta_{A}(x)\right\}+O\left(n^{-1 / 2}\right)$. If $z \in \partial A$, then since $f_{A}(z)$ is not defined, we let $\theta_{A}(z)$ be the average of $\theta_{A}(x)$ over all $x \in A$ (for which $f_{A}(x)$ is defined) with $|x-z|=1$. The Beurling estimate and a simple Harnack principle show that

$$
\theta_{A}(z)=\theta_{A}(x)+O\left(n^{-1 / 2}\right), \quad(x, z) \in \partial_{e} A .
$$

There are analogous Beurling-type results in the discrete case; the following is a corollary of $\left[11\right.$, Theorem 2.5.2]. Let $\tau_{A}=\min \left\{j \geq 0: S_{j} \notin A\right\}$.

Corollary 2.9 (Discrete Beurling Estimate). There is a constant $c<\infty$ such that if $r>0$, then $\mathbb{P}^{x}\left\{\left|S_{\tau_{A}}-x\right|>r \operatorname{dist}(x, \partial A)\right\} \leq c r^{-1 / 2}$.

In particular, if $|x|>n / 2$, the probability starting at $x$ of reaching $\mathbb{D}_{n / 2}$ before leaving $A$ is bounded above by $c n^{-1 / 2} \operatorname{dist}(x, \partial A)^{1 / 2}$. It is easy to show that $G_{A}(x) \leq c$ for $|x| \geq n / 4$; hence in this case we get

$$
G_{A}(x) \leq c n^{-1 / 2} \operatorname{dist}(x, \partial A)^{1 / 2}, \quad A \in \mathcal{A}^{n}, \quad|x| \geq n / 4
$$

Specifically, if $x \in \partial_{i} A$, then $G_{A}(x) \leq c n^{-1 / 2}$.

If $A \in \mathcal{A}$ and $0 \neq x \in \partial_{i} A$, then since $G_{A}(0)=G_{A \backslash\{x\}}(0)+\mathbb{P}\left\{\tau_{A}>\tau_{A \backslash\{x\}}\right\} G_{A}(x)$ it follows that

$$
G_{A}(0)=G_{A \backslash\{x\}}(0)+\frac{G_{A}(x)^{2}}{G_{A}(x, x)} .
$$

We can replace $A \backslash\{x\}$ in the above formula with the connected component of $A \backslash\{x\}$ containing the origin. In particular, if $A \in \mathcal{A}^{n}$ and $x \in \partial_{i} A$, then we conclude that $G_{A}(0)-$ $G_{A \backslash\{x\}}(0) \leq G_{A}(x)^{2} \leq c n^{-1}$.

\subsection{Excursion Poisson kernel}

Let $D$ be a domain in $\mathbb{C}$. We say that a connected $\Gamma$ is an (open) analytic arc of $\partial D$ if there is a domain $D^{\prime} \subset \mathbb{C}$ that is symmetric about the real axis and a conformal map $f: D^{\prime} \rightarrow f\left(D^{\prime}\right)$ such that $f\left(D^{\prime} \cap \mathbb{R}\right)=\Gamma$ and $f\left(D^{\prime} \cap \mathbb{H}\right)=f\left(D^{\prime}\right) \cap D$, where $\mathbb{H}$ denotes the upper half plane. We say that $\partial D$ is locally analytic at $x \in \partial D$, if there exists an analytic arc of $\partial D$ containing $x$. If $\Gamma$ is an analytic arc of $\partial D$ and $x, y \in \Gamma$, we write $x<y$ if $f^{-1}(x)<f^{-1}(y)$. We let $\mathbf{n}_{x}=\mathbf{n}_{x, D}$ be the unit normal at $x$ pointing into $D$.

If $x \in D$ and $\partial D$ is locally analytic at $y \in \partial D$, then both harmonic measure $\mathbb{P}^{x}\left\{B_{T_{D}} \in\right.$ $\mathrm{d} y\}$, and its density with respect to arc length, the Poisson kernel $H_{D}(x, y)$, are well-defined. Also, recall that for fixed $z \in D$, the function $y \mapsto H_{D}(z, y)$ is continuous in $y$, and that for fixed $y \in \partial D$, the function $z \mapsto H_{D}(z, y)$ is harmonic in $z$. If $x \in D$ and $\Gamma \subset \partial D$ is an analytic arc, then write

$$
H_{D}(x, \Gamma)=\int_{\Gamma} H_{D}(x, y)|\mathrm{d} y| .
$$

The Riemann mapping theorem and Lévy's theorem on the conformal invariance of Brownian motion [2] allow us to describe the behaviour of the Poisson kernel under a conformal transformation. 
Proposition 2.10. If $f: D \rightarrow D^{\prime}$ is a conformal transformation, $x \in D, \partial D$ is locally analytic at $y \in \partial D$, and $\partial D^{\prime}$ is locally analytic at $f(y)$, then $\mathbb{P}^{x}\left\{B_{T_{D}} \in \mathrm{d} y\right\}=\mathbb{P}^{f(x)}\left\{B^{\prime} T_{D^{\prime}} \in\right.$ $f(\mathrm{~d} y)\}$ where $B^{\prime}$ is a (time-change of) Brownian motion. Equivalently,

$$
H_{D}(x, y)=\left|f^{\prime}(y)\right| H_{D^{\prime}}(f(x), f(y)) .
$$

For each $\varepsilon>0$, let $\mu_{x, \varepsilon, D}$ denote the probability measure on paths obtained by starting a Brownian motion at $x+\varepsilon \mathbf{n}_{x}$ and stopping the path when it reaches $\partial D$. The excursion measure of $D$ at $x$ is defined by

$$
\mathcal{E}_{D}(x)=\lim _{\varepsilon \rightarrow 0+} \frac{1}{\varepsilon} \mu_{x, \varepsilon, D} .
$$

Excursion measure on $\Gamma$ is defined by

$$
\mathcal{E}_{D}(\Gamma)=\int_{\Gamma} \mathcal{E}_{D}(x)|\mathrm{d} x| .
$$

If $\Upsilon$ is another analytic arc on $\partial D$, we define $\mathcal{E}_{D}(\Gamma, \Upsilon)$ to be the excursion measure $\mathcal{E}_{D}(\Gamma)$ restricted to curves that end at $\Upsilon$ and whose endpoints are different. The excursion boundary measure is defined by $H_{\partial D}(\Gamma, \Upsilon)=\left|\mathcal{E}_{D}(\Gamma, \Upsilon)\right|$, where $|\cdot|$ denotes total mass. We can write

$$
H_{\partial D}(\Gamma, \Upsilon)=\int_{\Upsilon} \int_{\Gamma} H_{\partial D}(x, y)|\mathrm{d} x||\mathrm{d} y|,
$$

where $H_{\partial D}(x, y), x \neq y$, denotes the excursion Poisson kernel given by

$$
H_{\partial D}(x, y)=\lim _{\varepsilon \rightarrow 0+} \frac{1}{\varepsilon} H_{D}\left(x+\varepsilon \mathbf{n}_{x}, y\right)=\lim _{\varepsilon \rightarrow 0+} \frac{1}{\varepsilon} H_{D}\left(y+\varepsilon \mathbf{n}_{y}, x\right) .
$$

We can also write

$$
\mathcal{E}_{D}(\Gamma, \Upsilon)=\int_{\Upsilon} \int_{\Gamma} H_{\partial D}(x, y) \mathcal{E}_{D}^{\#}(z, w)|\mathrm{d} x||\mathrm{d} y|,
$$

where $\mathcal{E}_{D}^{\#}(z, w)=\mathcal{E}_{D}(z, w) / H_{\partial D}(x, y)$ is the excursion measure between $x$ and $y$ normalized to be a probability measure. We will consider $\mathcal{E}_{D}$ and $\mathcal{E}_{D}^{\#}$ as measures on curves modulo reparametrization. Conformal invariance of complex Brownian motion implies that $\mathcal{E}_{D}^{\#}$ is conformally invariant, i.e., if $f: D \rightarrow D^{\prime}$ is a conformal transformation, then $f \circ \mathcal{E}_{D}^{\#}(x, y)=$ $\mathcal{E}_{D^{\prime}}^{\#}(f(x), f(y))$.

Proposition 2.11. Suppose $f: D \rightarrow D^{\prime}$ is a conformal transformation and $x, y$ are distinct points on $\partial D$. Suppose that $\partial D$ is locally analytic at $x, y$ and $\partial D^{\prime}$ is locally analytic at $f(x)$, $f(y)$. Then $H_{\partial D}(x, y)=\left|f^{\prime}(x)\right|\left|f^{\prime}(y)\right| H_{\partial D^{\prime}}(f(x), f(y))$.

Proof. By definition, $H_{\partial D}(x, y)=\lim _{\varepsilon \rightarrow 0+} \varepsilon^{-1} H_{D}\left(x+\varepsilon \mathbf{n}_{x}, y\right)$. Therefore,

$$
\begin{aligned}
H_{\partial D}(x, y) & =\lim _{\varepsilon \rightarrow 0+} \varepsilon^{-1}\left|f^{\prime}(y)\right| H_{D^{\prime}}\left(f\left(x+\varepsilon \mathbf{n}_{x}\right), f(y)\right) \text { (Proposition 2.10) } \\
& =\left|f^{\prime}(y)\right| \lim _{\varepsilon \rightarrow 0+} \varepsilon^{-1} H_{D^{\prime}}\left(f(x)+\varepsilon f^{\prime}(x) \mathbf{n}_{x}+o(\varepsilon), f(y)\right) \\
& =\left|f^{\prime}(x)\right|\left|f^{\prime}(y)\right| \lim _{\varepsilon_{1} \rightarrow 0+} \varepsilon_{1}^{-1} H_{D^{\prime}}\left(f(x)+\varepsilon_{1} \mathbf{n}_{f(x)}+o\left(\varepsilon_{1}\right), f(y)\right) \\
& =\left|f^{\prime}(x)\right|\left|f^{\prime}(y)\right| H_{\partial D^{\prime}}(f(x), f(y))
\end{aligned}
$$

where we have written $\varepsilon_{1}=\varepsilon\left|f^{\prime}(x)\right|$, and have noted that $\mathbf{n}_{f(x)}=f^{\prime}(x) \mathbf{n}_{x} /\left|f^{\prime}(x)\right|$ and $\left|\mathbf{n}_{f(x)}\right|=\left|\mathbf{n}_{x}\right|=1$. 
Example 2.12. If $x=e^{i \theta}, y=e^{i \theta^{\prime}}, \theta \neq \theta^{\prime}$, then

$$
H_{\partial \mathbb{D}}(x, y)=\frac{1}{\pi} \frac{1}{|y-x|^{2}}=\frac{1}{2 \pi} \frac{1}{1-\cos \left(\theta^{\prime}-\theta\right)} .
$$

Example 2.13. If $\mathcal{R}_{L}=\{z: 0<R e(z)<L, 0<\operatorname{Im}(z)<\pi\}$, then separation of variables can be used to show that for $0<r<L, 0<q, q^{\prime}<\pi$,

$$
\begin{gathered}
H_{\mathcal{R}_{L}}\left(r+i q, L+i q^{\prime}\right)=\frac{2}{\pi} \sum_{n=1}^{\infty} \frac{\sinh (n r) \sin (n q) \sin \left(n q^{\prime}\right)}{\sinh (n L)} \\
H_{\partial \mathcal{R}_{L}}\left(i q, L+i q^{\prime}\right)=\frac{2}{\pi} \sum_{n=1}^{\infty} \frac{n \sin (n q) \sin \left(n q^{\prime}\right)}{\sinh (n L)}
\end{gathered}
$$

Example 2.14. In the Brownian excursion case, there is an exact form of Theorem 1.1. Suppose that $D \in \mathcal{D}$, and $x, y \in \partial D$ with $\partial D$ locally analytic at $x$ and $y$. Proposition 2.11 and Example 2.12 imply that $2 \pi H_{\partial D}(x, y)=\left|f^{\prime}(x)\right|\left|f^{\prime}(y)\right|\left(1-\cos \left(\theta_{D}(x)-\theta_{D}(y)\right)\right)^{-1}$ where $f \in$ $\mathcal{T}(D, \mathbb{D})$ with $f(0)=0$. However, Proposition 2.10, combined with the fact that harmonic measure from 0 in $\mathbb{D}$ is uniform on $\partial \mathbb{D}$, implies $\left|f^{\prime}(x)\right|^{-1} H_{D}(0, x)=\left|f^{\prime}(y)\right|^{-1} H_{D}(0, y)=$ $(2 \pi)^{-1}$. Hence, we conclude

$$
H_{\partial D}(x, y)=\frac{2 \pi H_{D}(0, x) H_{D}(0, y)}{1-\cos \left(\theta_{D}(x)-\theta_{D}(y)\right)} .
$$

We finish this subsection by stating a formula relating the excursion Poisson kernel and the Green's function for Brownian motion:

$$
H_{\partial D}(x, y)=\lim _{\varepsilon \rightarrow 0+} \frac{g_{D}\left(x+\varepsilon \mathbf{n}_{x}, y+\varepsilon \mathbf{n}_{y}\right)}{2 \pi \varepsilon^{2}}=\frac{\partial}{\partial \mathbf{n}_{x}} \frac{\partial}{\partial \mathbf{n}_{y}} g_{D}(x, y) .
$$

For the simply connected case, which is all that we will use, this follows from a straightforward computation. We omit the proof in the general case.

\subsection{Excursion Poisson kernel determinant}

Definition 2.15. If $D$ is a domain, and $x^{1}, \ldots, x^{k}, y^{1}, \ldots, y^{k}$ are distinct boundary points at which $\partial D$ is locally analytic, let $\mathbf{H}_{\partial D}(\mathbf{x}, \mathbf{y})=\left[H_{\partial D}\left(x^{i}, y^{j}\right)\right]_{1 \leq i, j \leq k}$ denote the $k \times k$ hitting matrix

$$
\mathbf{H}_{\partial D}(\mathbf{x}, \mathbf{y})=\left[\begin{array}{ccc}
H_{\partial D}\left(x^{1}, y^{1}\right) & \cdots & H_{\partial D}\left(x^{1}, y^{k}\right) \\
\vdots & \ddots & \vdots \\
H_{\partial D}\left(x^{k}, y^{1}\right) & \cdots & H_{\partial D}\left(x^{k}, y^{k}\right)
\end{array}\right]
$$

where $H_{\partial D}\left(x^{i}, y^{j}\right)$ is the excursion Poisson kernel, and $\mathbf{x}=\left(x^{1}, \ldots, x^{k}\right), \mathbf{y}=\left(y^{1}, \ldots, y^{k}\right)$.

A straightforward extension of Proposition 2.11 is that the determinant of the hitting matrix of excursion Poisson kernels is conformally covariant. 
Proposition 2.16. If $f: D \rightarrow D^{\prime}$ is a conformal transformation, $x^{1}, \ldots, x^{k}, y^{1}, \ldots, y^{k}$ are distinct points at which $\partial D$ is locally analytic, and $\partial D^{\prime}$ is locally analytic at $f\left(x^{1}\right), \ldots, f\left(x^{k}\right)$, $f\left(y^{1}\right), \ldots, f\left(y^{k}\right)$, then

$$
\operatorname{det} \mathbf{H}_{\partial D}(\mathbf{x}, \mathbf{y})=\left(\prod_{j=1}^{k}\left|f^{\prime}\left(x^{j}\right)\right|\left|f^{\prime}\left(y^{j}\right)\right|\right) \operatorname{det}\left[H_{\partial D^{\prime}}\left(f\left(x^{i}\right), f\left(y^{j}\right)\right)\right]_{1 \leq i, j \leq k} .
$$

Proof. By the definition of determinant,

$$
\begin{aligned}
& \operatorname{det} \mathbf{H}_{\partial D}(\mathbf{x}, \mathbf{y})=\sum_{\sigma}(\operatorname{sgn} \sigma) H_{\partial D}\left(x^{1}, y^{\sigma(1)}\right) \cdots H_{\partial D}\left(x^{k}, y^{\sigma(k)}\right) \\
& =\left(\prod_{j=1}^{k}\left|f^{\prime}\left(x^{j}\right)\right|\left|f^{\prime}\left(y^{j}\right)\right|\right) \sum_{\sigma}(\operatorname{sgn} \sigma) H_{\partial D^{\prime}}\left(f\left(x^{1}\right), f\left(y^{\sigma(1)}\right)\right) \cdots H_{\partial D^{\prime}}\left(f\left(x^{k}\right), f\left(y^{\sigma(k)}\right)\right) \\
& =\left(\prod_{j=1}^{k}\left|f^{\prime}\left(x^{j}\right)\right|\left|f^{\prime}\left(y^{j}\right)\right|\right) \operatorname{det}\left[H_{\partial D^{\prime}}\left(f\left(x^{i}\right), f\left(y^{j}\right)\right)\right]_{1 \leq i, j \leq k}
\end{aligned}
$$

where the sum is over all permutations $\sigma$ of $\{1, \ldots, k\}$, and $\operatorname{sgn} \sigma$ denotes the sign of the permutation.

It follows from this proposition that $\Lambda_{D}\left(x^{1}, \ldots, x^{k}, y^{k}, \ldots, y^{1}\right)$ given by (5) is a conformal invariant, and hence it can be defined for any Jordan domain by conformal invariance.

\subsection{Proof of Proposition 1.5}

By applying Proposition 2.16 and judiciously choosing $D=\mathcal{R}_{L}$, we now give the asymptotics as points gets close, and complete the proof of Proposition 1.5.

Proof of Proposition 1.5. Let $\mathbf{q}=\left(q_{1}, \ldots, q_{k}\right), \mathbf{q}^{\prime}=\left(q_{1}^{\prime}, \ldots, q_{k}^{\prime}\right)$,

$$
\vec{u}_{j}=\left[\begin{array}{c}
\sin \left(j q_{1}\right) \\
\sin \left(j q_{2}\right) \\
\vdots \\
\sin \left(j q_{k}\right)
\end{array}\right], \quad \vec{v}_{j}=\left[\begin{array}{c}
\sin \left(j q_{1}^{\prime}\right) \\
\sin \left(j q_{2}^{\prime}\right) \\
\vdots \\
\sin \left(j q_{k}^{\prime}\right)
\end{array}\right] .
$$

Using (25), we see that $(\pi / 2)^{k} \operatorname{det} \mathbf{H}_{\partial \mathcal{R}_{L}}\left(i \mathbf{q}, L+i \mathbf{q}^{\prime}\right)$ can be written as

$$
\operatorname{det}\left[\sum_{j=1}^{\infty} \frac{j \sin \left(j q_{1}\right)}{\sinh (j L)} \vec{v}_{j}, \sum_{j=1}^{\infty} \frac{j \sin \left(j q_{2}\right)}{\sinh (j L)} \vec{v}_{j}, \ldots, \sum_{j=1}^{\infty} \frac{j \sin \left(j q_{k}\right)}{\sinh (j L)} \vec{v}_{j}\right]_{1 \leq j \leq k} .
$$

By multilinearity of the determinant, we can write the determinant above as

$$
\sum_{j_{1}, \ldots, j_{k}} \frac{\left(j_{1} \cdots j_{k}\right) \sin \left(j_{1} q_{1}\right) \cdots \sin \left(j_{k} q_{k}\right)}{\sinh \left(j_{1} L\right) \cdots \sinh \left(j_{k} L\right)} \operatorname{det}\left[\vec{v}_{j_{1}}, \ldots, \vec{v}_{j_{k}}\right] .
$$


The determinants in the last sum equal zero if the indices are not distinct. Also it is not difficult to show that

$$
\sum_{j_{1}+\cdots+j_{k} \geq R} \frac{j_{1} \cdots j_{k}}{\sinh \left(j_{1} L\right) \cdots \sinh \left(j_{k} L\right)} \leq C(k, R) e^{-R L}
$$

Hence, except for an error of $O_{k}\left(e^{-\left(k^{2}+k+2\right) L / 2}\right)$, we see that $(\pi / 2)^{k} \operatorname{det} \mathbf{H}_{\partial \mathcal{R}_{L}}\left(i \mathbf{q}, L+i \mathbf{q}^{\prime}\right)$ equals

$$
k ! \sum_{\sigma} \frac{\sin \left(\sigma(1) q_{1}\right) \cdots \sin \left(\sigma(k) q_{k}\right)}{\sinh (L) \sinh (2 L) \cdots \sinh (k L)} \operatorname{det}\left[\vec{v}_{\sigma(1)}, \ldots, \vec{v}_{\sigma(k)}\right],
$$

where the sum is over all permutations $\sigma$ of $\{1, \ldots, k\}$. But

$$
\operatorname{det}\left[\vec{v}_{\sigma(1)}, \ldots, \vec{v}_{\sigma(k)}\right]=(\operatorname{sgn} \sigma) \operatorname{det}\left[\vec{v}_{1}, \ldots, \vec{v}_{k}\right] \text {. }
$$

Hence (27) equals

$$
\frac{k ! \operatorname{det}\left[\vec{u}_{1}, \ldots, \vec{u}_{k}\right] \operatorname{det}\left[\vec{v}_{1}, \ldots, \vec{v}_{k}\right]}{\sinh (L) \sinh (2 L) \cdots \sinh (k L)}
$$

which up to an error of $O_{k}\left(e^{-\left(k^{2}+k+2\right) L / 2}\right)$ equals $2^{k} k ! e^{-k(k+1) L / 2} \operatorname{det}\left[\vec{u}_{1}, \ldots, \vec{u}_{k}\right] \operatorname{det}\left[\vec{v}_{1}, \ldots, \vec{v}_{k}\right]$. To finish the proof, note that from (25), we can also write

$$
H_{\partial \mathcal{R}_{L}}\left(i q, L+i q^{\prime}\right)=\frac{4}{\pi} e^{-L} \sin q \sin q^{\prime}\left[1+O\left(e^{-L}\right)\right]
$$

so that

$$
(\pi / 2)^{k} \prod_{j=1}^{k} H_{\partial \mathcal{R}_{L}}\left(i q_{j}, L+i q_{j}^{\prime}\right)=2^{k} e^{-k L}\left(\prod_{j=1}^{k} \sin q_{j} \sin q_{j}^{\prime}\right)\left[1+O_{k}\left(e^{-L}\right)\right]
$$

\subsection{The discrete excursion Poisson kernel}

Definition 2.17. Suppose that $A$ is a proper subset of $\mathbb{Z}^{2}$, and let $\tau_{A}=\min \left\{j>0: S_{j} \in\right.$ $\partial A\}$. For $x \in A$ and $y \in \partial A$, define the discrete Poisson kernel (or hitting probability) to be $h_{A}(x, y)=\mathbb{P}^{x}\left\{S_{\tau_{A}}=y\right\}$.

We define the discrete analogue of the excursion Poisson kernel to be the probability that a random walk starting at $x \in \partial A$ takes its first step into $A$, and then exits $A$ at $y$.

Definition 2.18. Suppose that $A$ is a proper subset of $\mathbb{Z}^{2}$, and let $\tau_{A}=\min \left\{j>0: S_{j} \in\right.$ $\partial A\}$. For $x, y \in \partial A$, define the discrete excursion Poisson kernel to be $h_{\partial A}(x, y)=\mathbb{P}^{x}\left\{S_{\tau_{A}}=\right.$ $\left.y, S_{1} \in A\right\}$.

Note that

$$
h_{\partial A}(x, y)=\frac{1}{4} \sum_{(z, x) \in \partial_{e} A} h_{A}(z, y) .
$$

Also, a last-exit decomposition gives

$$
h_{A}(x, y)=\frac{1}{4} \sum_{(z, y) \in \partial_{e} A} G_{A}(x, z)
$$


where $G_{A}$ is the Green's function for simple random walk on $A$ as in (14). Hence,

$$
h_{\partial A}(x, y)=\frac{1}{16} \sum_{(z, y) \in \partial_{e} A} \sum_{(w, x) \in \partial_{e} A} G_{A}(w, z),
$$

which is a discrete analogue of (26).

\section{Proofs of the Main Results}

After briefly reviewing strong approximation and establishing some ancillary results in the first subsection, we devote the second subsection to the proof of Theorem 1.2 which relates the Green's function for Brownian motion to the Green's function for simple random walk in certain domains. While this result holds in general, it is most useful for points away from the boundary. In the final two subsections, we prove Theorem 1.1 by obtaining better estimates for the case of points near the boundary, provided they are not too close to each other. Throughout this section, suppose that $A \in \mathcal{A}^{n}$ with associated "union of squares" domain $\tilde{A} \in \mathcal{D}$. As in Section 2.1, let $f_{A} \in \mathcal{T}(\tilde{A}, \mathbb{D})$ with $f_{A}(0)=0, f_{A}^{\prime}(0)>0$, and recall from (13) that $f_{A}(x)=\exp \left\{-g_{A}(x)+i \theta_{A}(x)\right\}$, where $g_{A}$ is the Green's function for Brownian motion in $\tilde{A}$.

\subsection{Strong approximation}

In order to establish our Green's function estimates, we will need to establish a strong approximation result making use of the theorem of Komlós, Major, and Tusnády [7, 8]. Since we are concerned exclusively with complex Brownian motion and simple random walk on $\mathbb{Z}^{2}$, the results noted in [1] suffice.

Theorem 3.1 (Komlós-Major-Tusnády). There exist $c<\infty$ and a probability space $(\Omega, \mathcal{F}, \mathbb{P})$ on which are defined a two-dimensional Brownian motion $\hat{B}$ and a two-dimensional simple random walk $S$ with $\hat{B}_{0}=S_{0}$ such that for all $\lambda>0$ and each $n \in \mathbb{N}$,

$$
\mathbb{P}\left\{\max _{0 \leq t \leq n}\left|\frac{1}{\sqrt{2}} \hat{B}_{t}-S_{t}\right|>c(\lambda+1) \log n\right\}<c n^{-\lambda} .
$$

Here $S_{t}$ is defined for noninteger $t$ by linear interpolation. The one-dimensional proof may be found in [8], and the immediate extension to two dimensions is written down in [1, Lemma 3]. For our purposes, we will need to consider the maximum up to a random time, not just a fixed time. The following strong approximation will suffice. Our choice of $n^{8}$ is arbitrary and will turn out to be good enough.

Corollary 3.2 (Strong Approximation). There exist $C<\infty$ and a probability space $(\Omega, \mathcal{F}, \mathbb{P})$ on which are defined a two-dimensional Brownian motion $\hat{B}$ and a two-dimensional simple random walk $S$ with $\hat{B}_{0}=S_{0}$ such that

$$
\mathbb{P}\left\{\max _{0 \leq t \leq \sigma_{n}}\left|\frac{1}{\sqrt{2}} \hat{B}_{t}-S_{t}\right|>C \log n\right\}=O\left(n^{-10}\right),
$$

where $\sigma_{n}^{1}=\inf \left\{t:\left|S_{t}-S_{0}\right| \geq n^{8}\right\}, \sigma_{n}^{2}=\inf \left\{t:\left|\hat{B}_{t}-\hat{B}_{0}\right| \geq n^{8}\right\}$, and $\sigma_{n}=\sigma_{n}^{1} \vee \sigma_{n}^{2}$. 
Proof. If we choose $\rho>0$ such that $\mathbb{P}\left\{\left|\hat{B}_{1}\right| \geq 2\right\} \geq \rho$ and $\mathbb{P}\left\{\left|S_{n}\right| \geq 2 \sqrt{n}\right\} \geq \rho$ for all $n$ sufficiently large, then iteration shows that $\mathbb{P}\left\{\sigma_{n}>n^{36}\right\} \leq(1-\rho)^{n^{20}}=o\left(n^{-10}\right)$. Suppose that $\lambda=5 / 18$, and let $C=23 c / 18$ where $c$ is as in Theorem 3.1. Then, by using that theorem,

$\mathbb{P}\left\{\max _{0 \leq t \leq \sigma_{n}}\left|\frac{1}{\sqrt{2}} \hat{B}_{t}-S_{t}\right|>C \log n\right\} \leq \mathbb{P}\left\{\max _{0 \leq t \leq n^{36}}\left|\frac{1}{\sqrt{2}} \hat{B}_{t}-S_{t}\right|>C \log n\right\}+o\left(n^{-10}\right)=O\left(n^{-10}\right)$,

and the proof is complete.

Proposition 3.3. There exists a constant $c$ such that for every $n$, a Brownian motion $B$ and a simple random walk $S$ can be defined on the same probability space so that if $A \in \mathcal{A}^{n}$, $1<r \leq n^{20}$, and $x \in A$ with $|x| \leq n^{3}$, then

$$
\mathbb{P}^{x}\left\{\left|B_{T_{A}}-S_{\tau_{A}}\right| \geq c r \log n\right\} \leq c r^{-1 / 2} .
$$

Proof. For any given $n$, let $\hat{B}$ and $S$ be defined as in Corollary 3.2 above, and let $C$ be the constant in that corollary. Define $T_{A}^{\prime}=\inf \left\{t \geq 0: \operatorname{dist}\left(\hat{B}_{t} / \sqrt{2}, \partial \tilde{A}\right) \leq 2 C \log n\right\}$, $\tau_{A}^{\prime}=\inf \left\{t \geq 0: \operatorname{dist}\left(S_{t}, \partial A\right) \leq 2 C \log n\right\}, \hat{T}_{A}=\inf \left\{t \geq 0: \hat{B}_{t} / \sqrt{2} \in \partial A\right\}$ and consider the events

$$
\begin{gathered}
V_{1}=\left\{\sup _{0 \leq t \leq \sigma_{n}}:\left|\frac{1}{\sqrt{2}} \hat{B}_{t}-S_{t}\right|>C \log n\right\}, \quad V_{2}=\left\{\sup _{T_{A}^{\prime} \leq t \leq \hat{T}_{A}}\left|\hat{B}_{t}-\hat{B}_{T_{A}^{\prime}}\right| \geq r \log n\right\}, \\
\text { and } V_{3}=\left\{\sup _{\tau_{A}^{\prime} \leq t \leq \tau_{A}}\left|S_{t}-S_{\tau_{A}^{\prime}}\right| \geq r \log n\right\} .
\end{gathered}
$$

By the Beurling estimates (Corollaries 2.8 and 2.9), and the strong Markov property (applied separately to the random walk and the Brownian motion), it follows that $\mathbb{P}\left(V_{2} \cup V_{3}\right)=$ $O\left(r^{-1 / 2}\right)$, and from Corollary 3.2, $\mathbb{P}\left(V_{1}\right)=O\left(n^{-10}\right)=O\left(r^{-1 / 2}\right)$. Therefore, $\mathbb{P}\left(V_{1} \cup V_{2} \cup V_{3}\right)=$ $O\left(r^{-1 / 2}\right)$. Note that $\left|\left(\hat{B}_{\hat{T}_{A}} / \sqrt{2}\right)-S_{\tau_{A}}\right| \leq(r+2 C) \log n$ on the complement of $V_{1} \cup V_{2} \cup V_{3}$. Finally, if $B_{t}=\hat{B}_{2 t} / \sqrt{2}$, then $B_{t}$ is a standard Brownian motion and $B_{T_{A}}=\hat{B}_{\hat{T}_{A}} / \sqrt{2}$.

\subsection{Proof of Theorem 1.2}

Lemma 3.4. There exists a $c$ such that if $A \in \mathcal{A}^{n}$ and $|x| \leq n^{2}$, then

$$
\left|\mathbb{E}^{x}\left[\log \left|B_{T_{A}}\right|\right]-\mathbb{E}^{x}\left[\log \left|S_{\tau_{A}}\right|\right]\right| \leq c n^{-1 / 3} \log n .
$$

Proof. For any $n$, let $B$ and $S$ be as in Proposition 3.3, and let $V=V(n)$ be the event that $\left|B_{T_{A}}-S_{\tau_{A}}\right| \leq n^{2 / 3} \log n$. By that proposition, $\mathbb{P}(V) \geq 1-c n^{-1 / 3}$. Since $\operatorname{inrad}(A) \geq n$, we know that on the event $V$,

$$
|\log | B_{T_{A}}|-\log | S_{\tau_{A}}|| \leq c^{\prime} n^{-1 / 3} \log n .
$$

Note that $\mathbb{E}^{x}\left[\log \left|B_{T_{A}}\right| \cdot V^{c} \cdot\left\{\left|B_{T_{A}}\right| \leq n^{5}\right\}\right] \leq c \log n \mathbb{P}\left(V^{c}\right) \leq c n^{-1 / 3} \log n$. Using the Beurling estimates it is easy to see that

$$
\mathbb{E}^{x}\left[\log \left|B_{T_{A}}\right| .\left\{\left|B_{T_{A}}\right| \geq n^{5}\right\}\right]=O\left(n^{-1 / 3} \log n\right),
$$


and similarly for $\log \left|S_{\tau_{A}}\right|$ in the last two estimates. Hence,

$$
\mathbb{E}^{x}\left[\left(\log \left|B_{T_{A}}\right|+\log \left|S_{\tau_{A}}\right|\right) \cdot V^{c}\right] \leq c n^{-1 / 3} \log n .
$$

Proof of Theorem 1.2. First suppose $x \neq 0$. Recall from (11) that $g_{A}(x)=\mathbb{E}^{x}\left[\log \left|B_{T_{A}}\right|\right]-$ $\log |x|$ and from (15) that $G_{A}(x)=\mathbb{E}^{x}\left[a\left(S_{\tau_{A}}\right)\right]-a(x)$ with $a(x)$ as in (16). If $|x| \leq n^{2}$, then (1) follows from Lemma 3.4, and if $|x| \geq n^{2}$, then (1) follows directly from the bounds on $g_{A}(x)$ and $G_{A}(x)$ in (20) and (22), respectively.

If $x=0$, we can use the relation

$$
G_{A}(0)=1+G_{A}(e)=a(1)+G_{A}(e), \quad|e|=1,
$$

and $\left|f_{A}(e)\right|=\left|f_{A}^{\prime}(0)\right|+O\left(n^{-2}\right)$.

For any $A \in \mathcal{A}^{n}$, let $A^{*, n}$ be the set

$$
A^{*, n}=\left\{x \in A: g_{A}(x) \geq n^{-1 / 16}\right\} .
$$

The choice of 1/16 for the exponent is somewhat arbitrary, and slightly better estimates might be obtained by choosing a different exponent. However, since we do not expect the error estimate derived here to be optimal, we will just make this definition.

Corollary 3.5. If $A \in \mathcal{A}^{n}$, and $x \in A^{*, n}, y \in A$, then

$$
G_{A}(x, y)=\frac{2}{\pi} g_{A}(x, y)+k_{y-x}+O\left(n^{-7 / 24} \log n\right) .
$$

Proof. It follows from the Beurling estimates (20) and (22) that if $A \in \mathcal{A}^{n}$ and $x \in A^{*, n}$, then $\operatorname{dist}(x, \partial A) \geq c n^{7 / 8}$. That is, if we translate $A$ to make $x$ the origin, then the inradius of the translated set is at least $c n^{7 / 8}$. Hence, we can use Theorem 1.2 to deduce (30).

\subsection{An estimate for hitting the boundary}

Suppose that $A$ is any finite, connected subset of $\mathbb{Z}^{2}$, not necessarily simply connected, and let $V \subset \partial A$ be non-empty. Recall that $\tilde{A} \subset \mathbb{C}$ is the "union of squares" domain associated to $A$ as in Section 2.1. For every $y \in V$, consider the collection of edges containing $y$, namely

$\mathcal{E}_{y}=\left\{(x, y) \in \partial_{e} A\right\}$, and set $\mathcal{E}_{V}=\bigcup_{y \in V} \mathcal{E}_{y}$. If $\ell_{x, y}$ is the perpendicular line segment of length one intersecting $(x, y)$ in the midpoint as in Section 2.1, then define $\tilde{V}=\bigcup_{(x, y) \in \mathcal{E}_{V}} \ell_{x, y}$ to be the associated boundary arc in $\partial \tilde{A}$. Suppose that $\tau_{A}=\min \left\{j: S_{j} \in \partial A\right\}, T_{A}=T_{\tilde{A}}=$ $\inf \left\{t: B_{t} \in \partial \tilde{A}\right\}$, and throughout this subsection, write

$$
h(x)=h_{A}(x, V)=\mathbb{P}^{x}\left\{S_{\tau_{A}} \in V\right\}=\sum_{y \in V} h_{A}(x, y),
$$

and

$$
H(x)=H_{\tilde{A}}(x, \tilde{V})=\mathbb{P}^{x}\left\{B_{T_{A}} \in \tilde{V}\right\}=\int_{\tilde{V}} H_{\tilde{A}}(x, y)|\mathrm{d} y|,
$$

where $h_{A}$ and $H_{\tilde{A}}$ are the discrete Poisson kernel and the Poisson kernel, respectively. 
Definition 3.6. If $F: \mathbb{Z}^{2} \rightarrow \mathbb{R}$, let $L$ denote the discrete Laplacian defined by

$$
L F(x)=\mathbb{E}^{x}\left[F\left(S_{1}\right)-F\left(S_{0}\right)\right]=\frac{1}{4} \sum_{|e|=1}(F(x+e)-F(x)) .
$$

Call a function $F$ discrete harmonic at $x$ if $L F(x)=0$. If $L F(x)=0$ for all $x \in A \subseteq \mathbb{Z}^{2}$, then $F$ is called discrete harmonic in $A$.

Let $\Delta$ denote the usual Laplacian in $\mathbb{C}$, and recall that $F: \mathbb{C} \rightarrow \mathbb{R}$ is harmonic at $x$ if $\Delta F(x)=0$. Note that $L$ is a natural discrete analogue of $\Delta$. If $r>1, F: \mathbb{C} \rightarrow \mathbb{R}$, and $\Delta F(x)=0$ for all $x \in \mathbb{C}$ with $|x|<r$, then Taylor's series and uniform bounds on the derivatives of harmonic functions [2] imply that

$$
|L F(0)| \leq\|F\|_{\infty} O\left(r^{-3}\right) .
$$

Note that $h$ defined by (31) is discrete harmonic in $A$, and $H$ defined by (32) is harmonic in $\tilde{A}$. Our goal in the remainder of this subsection is to prove the following proposition.

Proposition 3.7. For every $\varepsilon>0$, there exists $a \delta>0$ such that if $A$ is a finite connected subset of $\mathbb{Z}^{2}, V \subset \partial A$, and $x \in A$ with $H(x) \geq \varepsilon$, then $h(x) \geq \delta$.

We first note that for every $n<\infty$, there is a $\delta^{\prime}=\delta^{\prime}(n)>0$ such that the proposition holds for all $A$ of cardinality at most $n$ and all $\varepsilon>0$. This is because $h$ and $H$ are strictly positive (since $V \neq \emptyset$ ) and the collection of connected subsets of $\mathbb{Z}^{2}$ containing the origin of cardinality at most $n$ is finite. Hence, we can choose

$$
\delta^{\prime}(n)=\min \mathbb{P}^{x}\left\{S_{\tau_{A}}=y\right\}
$$

where the minimum is over all finite connected $A$ of cardinality at most $n$, all $x \in A$, and all $y \in \partial A$. We now extend this to all $A$ for $x$ near the boundary.

Lemma 3.8. For every $\varepsilon>0, M<\infty$, there exists $a \delta>0$, such that if $A$ is a finite connected subset of $\mathbb{Z}^{2}, V \subset \partial A$, and $x \in A$ with $H(x) \geq \varepsilon$ and $\operatorname{dist}(x, \partial \tilde{A}) \leq M$, then $h(x) \geq \delta$.

Proof. By the recurrence of planar Brownian motion, we can find an $N=N(M, \varepsilon)$ such that $\mathbb{P}^{x}\left\{\operatorname{diam}\left(B\left[0, T_{A}\right]\right) \leq N\right\} \geq 1-(\varepsilon / 2)$ whenever $\operatorname{dist}(x, \partial \tilde{A}) \leq M$. Hence, if $\mathbb{P}^{x}\left\{B_{T_{A}} \in \tilde{V}\right\} \geq \varepsilon$ and $\operatorname{dist}(x, \partial \tilde{A}) \leq M$, then

$$
\mathbb{P}^{x}\left\{B_{T_{A}} \in \tilde{V} ; \operatorname{diam}\left(B\left[0, T_{A}\right]\right) \leq N\right\} \geq \varepsilon / 2,
$$

and the lemma holds with $\delta=\delta^{\prime}(3 N)$, say, where $\delta^{\prime}$ is defined as in (34) above.

For every $M<\infty$ and finite $A \subset \mathbb{Z}^{2}$, let

$$
\sigma_{M}=\sigma_{M, A}=\min \left\{j \geq 0: \operatorname{dist}\left(S_{j}, \partial A\right) \leq M\right\} .
$$

Since $A$ is finite and $h$ is a discrete harmonic function on $A$, it is necessarily bounded so that $h\left(S_{n \wedge \sigma_{M}}\right)$ is a bounded martingale. It then follows from the optional sampling theorem that $h(x)=\mathbb{E}^{x}\left[h\left(S_{\sigma_{M}}\right)\right]$ for all $x \in A$ since $\sigma_{M}$ is an a.s. finite stopping time. The next lemma gives a bound on the error in this equation if we replace $h$ with $H$. 
Lemma 3.9. For every $\varepsilon>0$, there exists an $M<\infty$ such that if $A$ is a finite connected subset of $\mathbb{Z}^{2}, V \subset \partial A$, and $x \in A$, then $\left|H(x)-\mathbb{E}^{x}\left[H\left(S_{\sigma_{M}}\right)\right]\right| \leq \varepsilon$.

Proof. For any function $F$ on $A$ and any $x \in A$,

$$
F(x)=\mathbb{E}^{x}\left[F\left(S_{\sigma_{M}}\right)\right]-\mathbb{E}^{x}\left[\sum_{j=0}^{\sigma_{M}-1} L F\left(S_{j}\right)\right] .
$$

(Note that $F$ is bounded since $A$ is finite.) Applying (35) to $H$ gives

$$
\left|H(x)-\mathbb{E}^{x}\left[H\left(S_{\sigma_{M}}\right)\right]\right| \leq \sum_{\operatorname{dist}(y, \partial A) \geq M} G_{A}(x, y)|L H(y)| .
$$

Since $H$ is harmonic and bounded by 1 , (33) implies $|L H(y)| \leq c \operatorname{dist}(y, \partial A)^{-3}$. A routine estimate shows that there is a constant $c$ such that for all $A \in \mathcal{A}, x \in A$, and $r \geq 1$, if a simple random walk is within distance $r$ of the boundary, then the probability that it will hit the boundary in the next $r^{2}$ steps is bounded below by $c / \log r$. Consequently, we have

$$
\sum_{r \leq \operatorname{dist}(y, \partial A) \leq 2 r} G_{A}(x, y) \leq c r^{2} \log r
$$

so that combining these estimates gives

$$
\sum_{r \leq \operatorname{dist}(y, \partial A) \leq 2 r} G_{A}(x, y)|L H(y)| \leq c r^{-1} \log r .
$$

It now follows that

$$
\begin{aligned}
\sum_{\operatorname{dist}(y, \partial A) \geq M} G_{A}(x, y)|L H(y)| & \leq \sum_{k: 2^{k-1} \geq M} \sum_{2^{k} \leq \operatorname{dist}(y, \partial A) \leq 2^{k+1}} G_{A}(x, y)|L H(y)| \\
& \leq c \sum_{k: 2^{k-1} \geq M} 2^{-k} \log \left(2^{k}\right) \\
& \leq C M^{-1} \log M .
\end{aligned}
$$

The proof is completed by choosing $M$ sufficiently large which will guarantee that the right side of (36) is smaller than $\varepsilon$.

Proof of Proposition 3.7. Fix $\varepsilon>0$, and suppose $H(x) \geq \varepsilon$. By the Lemma 3.9, we can find an $M=M(\varepsilon)$ such that

$$
\mathbb{E}^{x}\left[H\left(S_{\sigma_{M}}\right)\right]=\sum_{\operatorname{dist}(y, \partial A) \leq M} J(x, y) H(y) \geq \varepsilon / 2,
$$

where $J(x, y)=J_{A}(x, y ; M)=\mathbb{P}^{x}\left\{S_{\sigma_{M}}=y\right\}$. Hence

$$
\sum_{H(y) \geq \varepsilon / 4, \operatorname{dist}(y, \partial A) \leq M} J(x, y) \geq \sum_{H(y) \geq \varepsilon / 4, \operatorname{dist}(y, \partial A) \leq M} J(x, y) H(y) \geq \varepsilon / 4 .
$$

By Lemma 3.8, there is a $c=c(\varepsilon, M)$ such that $h(y) \geq c$ if $H(y) \geq \varepsilon / 4$ and $\operatorname{dist}(y, \partial A) \leq M$. Hence,

$$
h(x)=\sum_{\operatorname{dist}(y, \partial A) \leq M} J(x, y) h(y) \geq c \varepsilon / 4 .
$$




\subsection{The main estimates}

Theorem 1.2 with Corollary 3.5 give good estimates if $f_{A}(x)$ and $f_{A}(y)$ are not close to $\partial \mathbb{D}$. While the result is true even for points near the boundary, it is not very useful because the error terms are much larger than the value of the Green's function. Indeed, if $A \in \mathcal{A}^{n}$ and $x \in \partial_{i} A$, then $g_{A}(x)=O\left(n^{-1 / 2}\right)$ and $G_{A}(x)=O\left(n^{-1 / 2}\right)$, but $O\left(n^{-1 / 2}\right) \ll O\left(n^{-7 / 24} \log n\right)$, the error term in Corollary 3.5.

In this subsection we establish Proposition 3.10 which gives estimates for $x$ and $y$ close to the boundary provided that they are not too close to each other. Theorem 1.1 follows immediately. Recall that $A^{*, n}=\left\{x \in A: g_{A}(x) \geq n^{-1 / 16}\right\}$ as in (29).

The following estimates can be derived easily from (12). If $z=f_{A}(x)=(1-r) e^{i \theta}$, $z^{\prime}=f_{A}(y) \in \mathbb{D}$ with $\left|z-z^{\prime}\right| \geq r$, then

$$
g_{A}(x, y)=g_{\mathbb{D}}\left(z, z^{\prime}\right)=\frac{g_{\mathbb{D}}(z)\left(1-\left|z^{\prime}\right|^{2}\right)}{\left|z^{\prime}-e^{i \theta}\right|^{2}}\left[1+O\left(\frac{r}{\left|z-z^{\prime}\right|}\right)\right] .
$$

Similarly, if $z^{\prime}=f_{A}(y)=\left(1-r^{\prime}\right) e^{i \theta^{\prime}}$ with $r \geq r^{\prime}$ and $\left|z-z^{\prime}\right| \geq r$,

$$
g_{A}(x, y)=g_{\mathbb{D}}\left(z, z^{\prime}\right)=\frac{g_{\mathbb{D}}(z) g_{\mathbb{D}}\left(z^{\prime}\right)}{1-\cos \left(\theta-\theta^{\prime}\right)}\left[1+O\left(\frac{r}{\left|\theta-\theta^{\prime}\right|}\right)\right] .
$$

Proposition 3.10. Suppose $A \in \mathcal{A}^{n}$. If $x \in A \backslash A^{*, n}$ and

$$
J_{x, n}=\left\{z \in A:\left|f_{A}(z)-\exp \left\{i \theta_{A}(x)\right\}\right| \geq n^{-1 / 16} \log ^{2} n\right\},
$$

then for $y \in J_{x, n}$,

$$
\begin{gathered}
G_{A}(x, y)=G_{A}(x) \frac{1-\left|f_{A}(y)\right|^{2}}{\mid f_{A}(y)-e^{\left.i \theta_{A}(x)\right|^{2}}}\left[1+O\left(\frac{n^{-1 / 16} \log n}{\left|f_{A}(y)-e^{i \theta_{A}(x)}\right|}\right)\right], \quad y \in A^{*, n}, \\
G_{A}(x, y)=\frac{(\pi / 2) G_{A}(x) G_{A}(y)}{1-\cos \left(\theta_{A}(x)-\theta_{A}(y)\right)}\left[1+O\left(\frac{n^{-1 / 16} \log n}{\left|\theta_{A}(y)-\theta_{A}(x)\right|}\right)\right], \quad y \in A \backslash A^{*, n} .
\end{gathered}
$$

Thus, in view of the estimates (37) and (38) above, there is nothing surprising about the leading terms in (40) and (41). Proposition 3.10 essentially says that these relations are valid, at least in the dominant term, if we replace $g_{A}$ with $(\pi / 2) G_{A}$. Theorem 1.1 follows from this proposition and (28).

The hardest part of the proof is a lemma that states that if the random walk starts at a point $x$ with $f_{A}(x)$ near $\partial \mathbb{D}$, then, given that the walk does not leave $A, f_{A}\left(S_{j}\right)$ moves a little towards the centre of the disk before its argument changes too much.

Lemma 3.11. For $A \in \mathcal{A}^{n}$, let $\eta=\eta(A, n)=\min \left\{j \geq 0: S_{j} \in A^{*, n} \cup A^{c}\right\}$. There exist constants $c, \alpha$ such that if $A \in \mathcal{A}^{n}, x \in A \backslash A^{*, n}$, and $r>0$, then

$$
\begin{gathered}
\mathbb{P}^{x}\left\{\max _{0 \leq j \leq \eta-1}\left|f_{A}\left(S_{j}\right)-f_{A}(x)\right| \geq r n^{-1 / 16}\right\} \leq c e^{-\alpha r} \mathbb{P}^{x}\left\{S_{\eta} \in A^{*, n}\right\}, \\
\mathbb{P}^{x}\left\{\left|f_{A}\left(S_{\eta}\right)-f_{A}(x)\right| \geq r n^{-1 / 16} \mid S_{\eta} \in A^{*, n}\right\} \leq c e^{-\alpha r} .
\end{gathered}
$$


In particular, there is a $c_{0}$ such that if

$$
\xi=\xi\left(A, n, c_{0}\right)=\min \left\{j \geq 0: S_{j} \notin A \text { or }\left|f_{A}\left(S_{j}\right)-f_{A}(x)\right| \geq c_{0} n^{-1 / 16} \log n\right\},
$$

then

$$
\mathbb{P}^{x}\{\xi<\eta\} \leq c_{0} n^{-5} \mathbb{P}^{x}\left\{S_{\eta} \in A^{*, n}\right\} .
$$

In order to prove this lemma, we will need to establish several ancillary results. Therefore, we devote Section 3.4.1 which follows to the complete proof of this lemma, and then prove Proposition 3.10 in the separate Section 3.4.2.

\subsubsection{Proof of Lemma 3.11}

If $A \in \mathcal{A}$ and $x \in A$, let $d_{A}(x)$ be the distance from $f_{A}(x)$ to the unit circle. Note that $d_{A}(x)=\operatorname{dist}\left(f_{A}(x), \partial \mathbb{D}\right)=1-\left|f_{A}(x)\right|=1-\exp \left\{-g_{A}(x)\right\}$ in view of (13). As a first step in proving Lemma 3.11, we need the following.

Lemma 3.12. There exist constants $c, c^{\prime}, c^{\prime \prime}$, $\varepsilon$ such that if $A \in \mathcal{A}, x \in A$ with $d_{A}(x) \leq c$, and $\sigma=\sigma\left(x, A, c, c^{\prime}\right)$ is defined by

$$
\sigma=\min \left\{j \geq 0: S_{j} \notin A, d_{A}\left(S_{j}\right) \geq(1+c) d_{A}(x), \text { or }\left|\theta_{A}\left(S_{j}\right)-\theta_{A}(x)\right| \geq c^{\prime} d_{A}(x)\right\},
$$

then $\mathbb{P}^{x}\left\{S_{\sigma} \notin A\right\} \geq \varepsilon, \mathbb{P}^{x}\left\{S_{\sigma} \in A ; d_{A}\left(S_{\sigma}\right) \geq(1+c) d_{A}(x)\right\} \geq \varepsilon$, and

$$
\mathbb{P}^{x}\left\{\left|\theta_{A}\left(S_{\sigma}\right)-\theta_{A}(x)\right| \leq c^{\prime \prime} d_{A}(x) \mid S_{\sigma} \in A\right\}=1 .
$$

Remark. (42) is not completely obvious since the random walk takes discrete steps.

Proof. We start by stating three inequalities whose verification we leave to the reader. These are simple estimates for conformal maps on domains that are squares or the unions of two squares. Recall that $\mathcal{S}_{x}$ is the closed square of side one centred at $x$ whose sides are parallel to the coordinate axes. There exists a constant $c_{2} \in(1, \infty)$ such that if $A \in \mathcal{A} ; x, y, w \in A$; $x \neq y$; and $|w-x|=1$, then $d_{A}(z) \leq c_{2} d_{A}(x)$ for $z \in \mathcal{S}_{x} ;\left|f_{A}(z)-f_{A}(x)\right| \leq c_{2}\left|f_{A}\left(z^{\prime}\right)-f_{A}(x)\right|$ for $z, z^{\prime} \in \mathcal{S}_{y}$; and $\left|f_{A}(w)-f_{A}(x)\right| \leq c_{2} d_{A}(x)$. The first of these inequalities implies that if $z \in \mathcal{S}_{y}$ and $d_{A}(z) \geq 3 c_{2} d_{A}(x)$, then $d_{A}(y) \geq 3 d_{A}(x)$. Fix $A \in \mathcal{A}, x \in A$ with $d_{A}(x) \leq$ $1 /\left(100 c_{2}^{2}\right)$, and let

$$
J=J(x, A)=\left\{y \in A: \mathcal{S}_{y} \cap\left\{z \in \tilde{A}:\left|f_{A}(z)-f_{A}(x)\right|<5 c_{2} d_{A}(x)\right\} \neq \emptyset\right\} .
$$

That is, $y \in J$ if there is a $z \in \mathcal{S}_{y}$ with $\left|f_{A}(z)-f_{A}(x)\right|<5 c_{2} d_{A}(x)$. Note that $J$ is a connected subset of $A$ (although it is not clear whether it is simply connected) and $\tilde{J} \subseteq\{z \in \tilde{A}$ : $\left.\left|f_{A}(z)-f_{A}(x)\right|<5 c_{2}^{2} d_{A}(x)\right\}$. In particular, $d_{A}(y) \leq 6 c_{2}^{2} d_{A}(x)$ and $\left|\theta_{A}(y)-\theta_{A}(x)\right|<c^{\prime} d_{A}(x)$ for all $y \in J$.

There is a positive probability $\rho_{1}$ that a Brownian motion in $\mathbb{D}$ starting at $f_{A}(x)$ leaves $\mathbb{D}$ before leaving the disk of radius $2 d_{A}(x)$ about $f_{A}(x)$. By conformal invariance, this implies that with probability at least $\rho_{1}$, a Brownian motion starting at $x$ leaves $\tilde{J}$ at $\partial \tilde{A}$. Hence by Proposition 3.7, there is an $\varepsilon_{1}$ such that $\mathbb{P}^{x}\left\{S_{\tau_{J}} \notin A\right\} \geq \varepsilon_{1}$. 
Similarly, there is a positive probability $\rho_{2}$ that a Brownian motion in the disk starting at $f_{A}(x)$ reaches $\left[1-6 c_{2}^{2} d_{A}(x)\right] \mathbb{D}$ before leaving $\mathbb{D}$ and before leaving the set $\left\{z: \mid \theta_{A}(z)-\right.$ $\left.\theta_{A}(x) \mid \leq d_{A}(x)\right\}$. Note that $d_{A}(z)^{2} \geq\left|f_{A}(z)-f_{A}(x)\right|^{2}-d_{A}(x)^{2}$ on this set. In particular, with probability at least $\rho_{2}$, a Brownian motion starting at $x$ leaves $\tilde{J}$ at a point $z$ with $d_{A}(z) \geq 4 c_{2} d_{A}(x)$. Such a point $z$ must be contained in an $\mathcal{S}_{y}$ with $d_{A}(y) \geq 4 d_{A}(x)$. Hence, again using Proposition 3.7, there is a positive probability $\varepsilon_{2}$ that a random walk starting at $x$ reaches a point $y \in J$ with $d_{A}(y) \geq 4 d_{A}(x)$ before leaving $J$. In the notation of Lemma 3.12 choose $c=1 /\left(100 c_{2}^{2}\right)$, let $c^{\prime}$ be the $c^{\prime}$ mentioned above, and let $\varepsilon=\min \left\{\varepsilon_{1}, \varepsilon_{2}\right\}$. Then we have already shown that $\mathbb{P}^{x}\left\{S_{\sigma} \notin A\right\} \geq \varepsilon$ and $\mathbb{P}^{x}\left\{S_{\sigma} \in A ; d_{A}\left(S_{\sigma}\right) \geq(1+c) d_{A}(x)\right\} \geq \varepsilon$. Also, if $y, w \in A$ with $|y-w|=1, d_{A}(y) \leq d_{A}(x)$, and $\left|\theta_{A}(y)-\theta_{A}(x)\right| \leq c^{\prime} d_{A}(x)$, then

$$
\left|f_{A}(w)-f_{A}(x)\right| \leq\left|f_{A}(w)-f_{A}(y)\right|+\left|f_{A}(y)-f_{A}(x)\right| \leq c^{\prime \prime \prime} d_{A}(x),
$$

which implies that $\left|\theta_{A}(w)-\theta_{A}(x)\right| \leq c^{\prime \prime} d_{A}(x)$ for an appropriate $c^{\prime \prime}$. This gives the last assertion in Lemma 3.12 and completes the proof.

Corollary 3.13. There exist constants $c, c^{\prime}, \alpha$ such that if $a \in(0,1 / 2), A \in \mathcal{A}$, and $x \in A$ with $d_{A}(x)<a$, then the probability that a random walk starting at $x$ reaches the set $\left\{y \in A: d_{A}(y) \geq a\right\}$ without leaving the set $\left\{y \in A:\left|\theta_{A}(y)-\theta_{A}(x)\right| \leq c^{\prime} a\right\}$ is at least $c\left(d_{A}(x) / a\right)^{\alpha}$.

Proof. Let $Q(r, a, b)$ be the infimum over all $A \in \mathcal{A}$ and $x \in A$ with $d_{A}(x) \geq r$ of the probability that a random walk starting at $x$ reaches the set $\left\{y \in A: d_{A}(y) \geq a\right\}$ without leaving the set $\left\{y \in A:\left|\theta_{A}(y)-\theta_{A}(x)\right| \leq b\right\}$. It follows from Lemma 3.12 that there exist $q>0, \rho<1$, and a $c^{\prime \prime}$ such that $Q\left(\rho^{k}, \rho^{j}, b\right) \geq q Q\left(\rho^{k-1}, \rho^{j}, b-c^{\prime \prime} \rho^{k}\right)$. By iterating this we get $Q\left(\rho^{k}, \rho^{j}, 2 c^{\prime \prime}(1-\rho)^{-1} \rho^{j}\right) \geq q^{k-j}$. This and obvious monotonicity properties give the result.

Remark. A similar proof gives an upper bound of $c_{1}\left(d_{A}(x) / a\right)^{\alpha_{1}}$, but this result is not needed.

For any $a \in(0,1)$ and any $\theta_{1}<\theta_{2}$, let $\eta\left(a, \theta_{1}, \theta_{2}\right)$ be the first time $t \geq 0$ that a random walk leaves the set $\left\{y \in A: d_{A}(y) \leq a, \theta_{1} \leq \theta_{A}(y) \leq \theta_{2}\right\}$. Let

$$
q\left(x, a, \theta_{1}, \theta_{2}\right)=\mathbb{P}^{x}\left\{d_{A}\left(S_{\eta\left(a, \theta_{1}, \theta_{2}\right)}\right)>a \mid S_{\eta\left(a, \theta_{1}, \theta_{2}\right)} \in A\right\},
$$

and note that if $\theta_{1} \leq \theta_{1}^{\prime} \leq \theta_{2}^{\prime} \leq \theta_{2}$, then $q\left(x, a, \theta_{1}^{\prime}, \theta_{2}^{\prime}\right) \leq q\left(x, a, \theta_{1}, \theta_{2}\right)$.

Proposition 3.14. There exist constants $c, c_{1}$ such that if $a \in(0,1 / 2), A \in \mathcal{A}$, and $x \in A$, then $q\left(x, a, \theta_{A}(x)-c_{1} a, \theta_{A}(x)+c_{1} a\right) \geq c$.

Proof. For every $r>0$ and $m \in \mathbb{N}$ let $h(m, r)=\inf q\left(x, a, \theta_{A}(x)-r a, \theta_{A}(x)+r a\right)$ where the infimum is taken over all $a \in(0,1 / 2), A \in \mathcal{A}$, and all $x \in A$ with $d_{A}(x) \geq 2^{-m} a$. The proposition is equivalent to saying that there is a $c_{1}$ such that

$$
\inf _{m} h\left(m, c_{1}\right)>0
$$

It follows from Corollary 3.13 that there is a $c^{\prime}$ such that $h\left(m, c^{\prime}\right)>0$ for each $m$; more specifically, there exist $c_{2}, \beta$ such that $h(m, r) \geq c_{2} e^{-\beta m}$ for $r \geq c^{\prime}$. 
Suppose $x \in A$ with $2^{-(m+1)} a \leq d_{A}(x)<2^{-m} a$. Start a random walk at $x$ and stopped at $t^{*}$, defined to be the first time $t$ when one of the following is satisfied: $S_{t} \notin A, d_{A}\left(S_{t}\right) \geq 2^{-m} a$, or $\left|\theta_{A}\left(S_{t}\right)-\theta_{A}(x)\right| \geq m^{2} 2^{-m} a$. By iterating Lemma 3.12, we see that the probability that the last of these three possibilities occurs is bounded above by $c^{\prime \prime} e^{-\beta^{\prime} m^{2}}$. Choose $M$ sufficiently large such that for $m \geq M$, the last term is less than $c_{2} e^{-2 \beta(m+1)}$, and such that

$$
\mathbb{P}^{x}\left\{\left|\theta_{A}\left(S_{t^{*}}\right)-\theta_{A}(x)\right| \leq m^{3} 2^{-m} a \mid S_{t^{*}} \in A\right\}=1 .
$$

Note that (42) shows (43) holds for all sufficiently large $m$; for such $m$, if $r \geq c^{\prime}$, then $h(m+1, r) \geq\left[1-e^{-\beta m}\right] h\left(m, r-m^{3} 2^{-m}\right)$. In particular, if

$$
r>c^{\prime}+\sum_{m=1}^{\infty} m^{3} 2^{-m}
$$

then

$$
h(m, r) \geq\left[\prod_{j=1}^{\infty}\left(1-e^{-\beta j}\right)\right] h\left(M, c^{\prime}\right)>0 .
$$

Corollary 3.15. There exist $c, \beta$ such that if $a \in(0,1 / 2), r>0, A \in \mathcal{A}$, and $x \in A$, then $q\left(x, a, \theta_{A}(x)-r a, \theta_{A}(x)+r a\right) \geq 1-c e^{-\beta r}$.

\subsubsection{Proof of Proposition 3.10}

Suppose $A \in \mathcal{A}^{n},(z, y) \in \partial_{e} A^{*, n}$, and let $\eta$ be as in Lemma 3.11. Since $g_{A}$ is harmonic in the disk of radius $O\left(n^{7 / 8}\right)$ about $z$, standard estimates for positive harmonic functions give

$$
\left|g_{A}(z)-g_{A}(y)\right| \leq O\left(n^{-7 / 8}\right) g_{A}(z) \leq o\left(n^{-7 / 8}\right) .
$$

Since $g_{A}(y)<n^{-1 / 16} \leq g_{A}(z)$, we conclude $g_{A}(z)=n^{-1 / 16}+o\left(n^{-7 / 8}\right)$, and similarly for $g_{A}(y)$. Hence, by Theorem 1.2,

$$
G_{A}(z)=(2 / \pi) n^{-1 / 16}+O\left(n^{-1 / 3} \log n\right)=(2 / \pi) n^{-1 / 16}\left[1+O\left(n^{-13 / 48} \log n\right)\right],
$$

and similarly for $G_{A}(y)$. Therefore, for any $x \in A \backslash A^{*, n}$,

$$
\begin{aligned}
G_{A}(x) & =\mathbb{P}^{x}\left\{S_{\eta} \in A^{*, n}\right\} \mathbb{E}^{x}\left[G_{A}\left(S_{\eta}\right) \mid S_{\eta} \in A^{*, n}\right] \\
& =(2 / \pi) n^{-1 / 16} \mathbb{P}^{x}\left\{S_{\eta} \in A^{*, n}\right\}\left[1+O\left(n^{-13 / 48} \log n\right)\right] .
\end{aligned}
$$

In a similar fashion, note that if $x, y \in A^{*, n}$, then $g_{A}(x, y) \geq c n^{-1 / 8}$, and hence by Corollary 3.5, if $|x-y| \geq n^{1 / 2}$, then $G_{A}(x, y)=(2 / \pi) g_{A}(x, y)\left[1+O\left(n^{-1 / 6} \log n\right)\right]$.

If $A \in \mathcal{A}^{n}, x \in A \backslash A^{*, n}$, and $y \in A^{*, n}$, then the strong Markov property gives

$$
G_{A}(x, y)=\sum_{z \in A^{*, n}} \mathbb{P}^{x}\left\{S_{\eta}=z\right\} G_{A}(z, y) .
$$

If $x \in A \backslash A^{*, n}$, let $R(x)=R(x, n, A)=\left\{z \in A:\left|f_{A}(z)-f_{A}(x)\right| \leq c_{0} n^{-1 / 16} \log n\right\}$, where $c_{0}$ is the constant in Lemma 3.11. As a consequence of that lemma we see that

$$
\sum_{z \in A^{*, n} \cap R(x)} \mathbb{P}^{x}\left\{S_{\eta}=z\right\}=\left[1-O\left(n^{-5}\right)\right] \sum_{z \in A^{*, n}} \mathbb{P}^{x}\left\{S_{\eta}=z\right\} .
$$


But $c n^{-1 / 8} \leq G_{A}(z, y) \leq c^{\prime} \log n$ for $z, y \in A^{*, n}$; hence,

$$
G_{A}(x, y)=\left[1+o\left(n^{-4}\right)\right] \sum_{z \in A^{*, n} \cap R(x)} \mathbb{P}^{x}\left\{S_{\eta}=z\right\} G_{A}(z, y) .
$$

If $\left|f_{A}(y)-f_{A}(x)\right| \geq n^{-1 / 16} \log ^{2} n$, and $z \in A^{*, n} \cap R(x)$, then from (37),

$$
g_{A}(z, y)=\frac{n^{-1 / 16}\left(1-\left|f_{A}(y)\right|^{2}\right)}{\left|f_{A}(y)-e^{i \theta_{A}(x)}\right|^{2}}\left[1+O\left(\frac{n^{-1 / 16} \log n}{\left|f_{A}(y)-e^{i \theta_{A}(x)}\right|}\right)\right] .
$$

Hence, using Corollary 3.5,

$$
G_{A}(x, y)=\mathbb{P}^{x}\left\{S_{\eta} \in A^{*, n}\right\} \frac{(2 / \pi) n^{-1 / 16}\left(1-\left|f_{A}(y)\right|^{2}\right)}{\left|f_{A}(y)-e^{i \theta_{A}(x)}\right|^{2}}\left[1+O\left(\frac{n^{-1 / 16} \log n}{\left|f_{A}(y)-e^{i \theta_{A}(x)}\right|}\right)\right] .
$$

Combining this with (44) gives (40). If $y \in \partial_{i} A^{*, n}$, then we can write

$$
G_{A}(x, y)=G_{A}(x) \frac{n^{-1 / 16}}{1-\cos \left(\theta_{A}(x)-\theta_{A}(y)\right)}\left[1+O\left(\frac{n^{-1 / 16} \log n}{\left|y-e^{i \theta_{A}(x)}\right|}\right)\right] .
$$

Now suppose $y \in J_{x, n} \backslash A^{*, n}$. Then we can write

$$
G_{A}(x, y)=G_{A \backslash A^{*, n}}(x, y)+\sum_{z \in A^{*, n}} \mathbb{P}^{x}\left\{S_{\eta}=z\right\} G_{A}(z, y),
$$

and using (40) on $G_{A}(z, y)$ gives $G_{A}(x, y) \geq c n^{-1 / 8} \mathbb{P}^{x}\left\{S_{\eta} \in A^{*, n}\right\} \mathbb{P}^{y}\left\{S_{\eta} \in A^{*, n}\right\}$. However, provided $R(x) \cap R(y)=\emptyset$, which is true for $n$ sufficiently large, Lemma 3.11 shows that $G_{A \backslash A^{*, n}}(x, y) \leq c n^{-10} \mathbb{P}^{x}\left\{S_{\eta} \in A^{*, n}\right\} \mathbb{P}^{y}\left\{S_{\eta} \in A^{*, n}\right\}$. Therefore,

$$
G_{A}(x, y)=\left[1+o\left(n^{-9}\right)\right] \sum_{z \in A^{*, n}} \mathbb{P}^{x}\left\{S_{\eta}=z\right\} G_{A}(z, y)
$$

and we can use (45) to deduce (41).

\section{Acknowledgements}

Much of this research was done by the first author in his Ph.D. dissertation [9] under the supervision of the second author. The first author expresses his gratitude to the second author for his continued guidance and support. Thanks are also owed to an anonymous referee for many valuable comments.

\section{References}

[1] P. Auer. Some hitting probabilities of random walks on $Z^{2}$. In I. Berkes, E. Csáki, and P. Révész, editors, Limit Theorems in Probability and Statistics, volume 57 of Colloquia Mathematica Societatis János Bolyai, pages 9-25. North-Holland, Budapest, Hungary, 1990. 
[2] R. F. Bass. Probabilistic Techniques in Analysis. Springer-Verlag, New York, NY, 1995.

[3] P. L. Duren. Univalent Functions, volume 259 of Grundlehren der mathematischen Wissenschaften. Springer-Verlag, New York, NY, 1983.

[4] S. Fomin. Loop-erased walks and total positivity. Trans. Amer. Math. Soc., 353:35633583, 2001.

[5] Y. Fukai and K. Uchiyama. Potential kernel for two-dimensional random walk. Ann. Probab., 24:1979-1992, 1996.

[6] R. Kenyon. The asymptotic determinant of the discrete Laplacian. Acta Math., 185:239$286,2000$.

[7] J. Komlós, P. Major, and G. Tusnády. An approximation of partial sums of independent RV'-s, and the sample DF. I. Z. Wahrscheinlichkeitstheorie verw. Gebiete, 32:111-131, 1975 .

[8] J. Komlós, P. Major, and G. Tusnády. An approximation of partial sums of independent RV's, and the sample DF. II. Z. Wahrscheinlichkeitstheorie verw. Gebiete, 34:33-58, 1976.

[9] M. J. Kozdron. Simple random walk excursion measure in the plane. Ph.D. dissertation, Duke University, Durham, NC, 2004.

[10] M. J. Kozdron. On the scaling limit of simple random walk excursion measure in the plane. Preprint, 2005. Available online at arXiv:math.PR/0506337.

[11] G. F. Lawler. Intersections of Random Walks. Birkhäuser, Boston, MA, 1991.

[12] G. F. Lawler. Loop-erased random walk. In M. Bramson and R. Durrett, editors, Perplexing Problems in Probability: Festschrift in Honor of Harry Kesten, volume 44 of Progress in Probability, pages 197-217. Birkhäuser, Boston, MA, 1999.

[13] G. F. Lawler. Conformally Invariant Processes in the Plane, volume 114 of Mathematical Surveys and Monographs. American Mathematical Society, Providence, RI, 2005.

[14] G. F. Lawler, O. Schramm, and W. Werner. Conformal invariance of planar loop-erased random walks and uniform spanning trees. Ann. Probab., 32:939-995, 2004.

[15] O. Schramm and S. Sheffield. The harmonic explorer and its convergence to SLE(4). Preprint, 2003. Available online at arXiv:math.PR/0310210.

[16] S. Smirnov. Critical percolation in the plane. Conformal invariance, Cardy's formula, scaling limits. C. R. Acad. Sci. Paris Sér. I Math., 333:239-244, 2001.

[17] D. Zhan. Stochastic Loewner evolution in doubly connected domains. Probab. Theory Related Fields, 129:340-380, 2004. 